\title{
New offset-free method for model predictive control of open channels
}

\author{
Klaudia Horváth ${ }^{\mathrm{a}, *}$, Eduard Galvis ${ }^{\mathrm{b}}$, Manuel Gómez Valentín ${ }^{\mathrm{a}}$, José Rodellar ${ }^{\mathrm{b}}$ \\ ${ }^{a}$ Technical University of Catalonia, Barcelonatech, Department of Hydraulic, Maritime and Environmental Engineering, Jordi Girona 1, Barcelona 08032, \\ Spain \\ ${ }^{\mathrm{b}}$ Technical University of Catalonia, Barcelonatech, Department of Applied Mathematics III, Jordi Girona 1, Barcelona 08034, Spain
}

\section{A R T I C L E I N F O}

Keywords:

Model predictive control

Offset-free

Irrigation

Automatic control

Integrator resonance

Experimental canal

\begin{abstract}
A B S T R A C T
Irrigation or drainage canals can be controlled by model predictive control (MPC). Applying MPC with an internal model in the presence of unknown disturbances in some cases can lead to steady state offset. Therefore an additional component should be implemented along with the MPC. A new method eliminating the offset has been developed in this paper for MPC. It is based on combining two basic approaches of MPC. It has been implemented to control water levels in the three-pool UPC laboratory canal and further numerically tested using a test case benchmark proposed by the American Society of Civil Engineers (ASCE). It has been found that the developed offset-free method is able to eliminate the steady-state offset, while taking into account known and unknown disturbances.
\end{abstract}

\section{Introduction}

Automatic control of delivery canals has been adopted in the last years with the purpose of improving the efficiency in the management of irrigation freshwater. The idea is to automatically manipulate structures, such as gates, pumps and others, in order to achieve a control objective, which can be stated in terms of discharges or water levels. Typical control operations involve setpoint changes in these variables, according to management policies, and maintenance of such setpoints in spite of the presence of disturbances. Most common disturbances are produced due to water offtakes from the controlled canal to secondary canals or to water users. These disturbances may be known, if offtakes are scheduled in time and quantity, or unknown.

One of the control methods used to control open channels is model predictive control (MPC) (Gómez, Rodellar, \& Mantecón, 2002; van Overloop, 2006; Igreja, Cadete, \& Lemos, 2011; Negenborn, van Overloop, Keviczky, \& de Schutter, 2009; van Overloop, Clemmens, Strand, Wagemaker, \& Bautista, 2010a; Zafra-Cabeza, Maestre, Ridao, Camacho, \& Sánchez, 2011; Lemos, Machado, Nogueira, Rato, \& Rijo, 2009; Aguilar, Langarita, Linares, \& Rodellar, 2009, 2012).

The term MPC refers to a family of control algorithms whose common property is having state and output predictions by using an internal model and carrying out an optimization using the present

\footnotetext{
* Corresponding author. Current address: Ecole des Mines de Douai, Department of Informatics and Automation, 764 Boulevard Lahure, 59500 Douai, France. E-mail address: hklau85@gmail.com (K. Horváth).
}

and future predicted data (Mosca, 1995; Martín Sánchez \& Rodellar, 1996; Camacho \& Bordons, 1998).

A predictive controller calculates a control action based on the difference between the existing and the predicted errors during a prediction horizon. It does not only act on the error at the first instant, but it prepares an action that would minimize the errors over the prediction horizon. If the internal predictive model was ideally correct, the controller would be able to drive the system exactly to the setpoint. However, if the model is different than the real process, or there are disturbances or noise that are not described by the model, the controller might not be able to achieve it. In particular, for constant offset-like disturbances, the controlled output could reach a steady state but with an undesired offset with respect to the setpoint. There are two main ways of eliminating the offset: (1) model the disturbances, or (2) extend the predictive controller with an integral action.

In the industry, the inclusion of disturbance models is a common prerequisite in any standard industrial MPC implementation (Venkat, Rawlings, \& Wright, 2006; Camacho \& Bordons, 1998) considering that the origin of the disturbances is known. Pannocchia and Rawlings (2003) and Badgwell and Muske (2002) simultaneously arrived at the same conclusions about disturbance models and deduced conditions for offset free tracking. These conditions are summarized in Borrelli and Morari (2007).

The disadvantage of disturbance models is the difficulty in tuning the observer, since very often the nature of the disturbance is unknown. Wang (2009) describes the use of a built-in integrator, but it can lead to instabilities in some cases. To solve this problem an exponential data weighting is proposed (Wang, 2001). The predictive control approach by Martín Sánchez and Rodellar (1996) proposes an 
incremental formulation, which is proved to cancel offsets for constant disturbances.

In the field of canal control, Begovich, Ruiz, Besançon, Aldana, and Georges (2007) use the internal model principle: in order to reject constant disturbances it is necessary that an integrator appears in the closed loop system, that is an internal model of the constant disturbance. Therefore they propose an augmented model, similar to that of Wang (2009), which contains a disturbance model based on integrators. The use of additional feedforward component in the control loop is described in Aguilar et al. (2009) in a predictive control scheme.

Weyer (2008) proposes a LQ regulator that can deal with known disturbances. In Cantoni et al. (2007) a feedforward term is added that also acts as a "decoupler".

In this work a new offset-free MPC is proposed based on the predictive controller developed by Rodellar, Gómez, and Bonet (1993). This controller has zero steady state offset but cannot handle known disturbances well. The basic idea is to combine the two controllers to achieve a control that can lead to offset-free result and able to handle known and unknown disturbances. The proposed controller has been implemented and tested numerically and experimentally on the laboratory canal of the Technical University of Catalonia (UPC-PAC) and numerically on the ASCE Test Canal 2. Apart from the proposed method, other four MPC methods have been implemented numerically for comparison purposes.

This paper is structured as follows. Section 2 describes the UPC-PAC laboratory facility and the ASCE Test Canal 2. Section 3 presents the modelling issues and Section 4 presents the control developments. In order to build an offset free controller, three steps are followed: first, a basic controller is discussed (Section 4.1); second, another controller is described with integral action (Section 4.2); and finally the new offset-free predictive control is derived (Section 4.3). Additionally, in Sections 4.4 and 4.5 two methods are revised from the literature that were developed to eliminate steady state offset and are implemented in this work for comparison purposes. The test cases are presented in Section 5 and experimental and numerical results are shown and discussed in Section 6. Finally the work is concluded (Section 7).

\section{Case studies}

\subsection{Laboratory canal}

The UPC-PAC canal (Canal de Prueba de Algoritmos de Control Universitat Politècnica de Catalunya) is specially designed to develop basic and applied research in the field of control of irrigation canals. The canal is $0.44 \mathrm{~m}$ wide and $220 \mathrm{~m}$ long and has zero slope.

In this work the canal is configured to have three pools (see Fig. 1), and each pool is separated with a motorized sluice gate. The gravity offtakes are located at the downstream end of each canal pool.

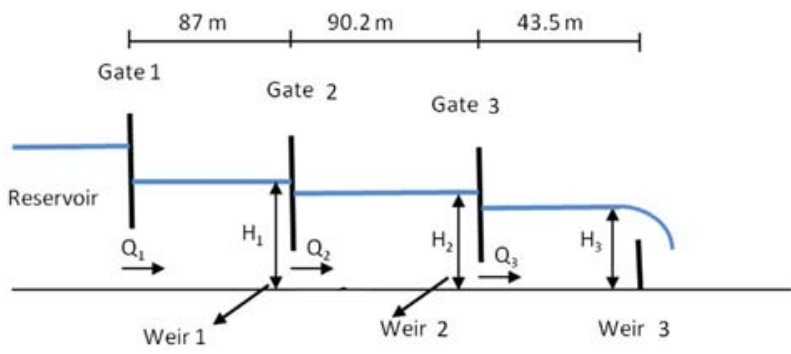

Fig. 1. Scheme of the three pool configuration of the UPC-PAC.
The SCADA system was developed in Matlab/Simulink environment, which allows the test of any control algorithm developed in Embedded Matlab language (Mathworks, 2008).

The UPC-PAC is short, completely affected by backwater. The low friction and the zero slope enhance the appearance of resonance waves. This phenomenon in the laboratory canal has been previously studied (Horváth, 2013; van Overloop, Horváth, \& Aydin, 2014). it is not presented in detail herein, since this paper deals with another problem. More information about the laboratory canal can be found in Sepúlveda (2008).

\subsection{The ASCE Test Canal 2}

Test Canal 2 has 8 canal pools and the control objective is to keep the downstream water levels at their setpoints by controlling the gate openings in the system. Gravity offtakes are located at the downstream end of each pool. The geometry of the canal and the details of the tests can be found in Clemmens, Kacerek, Grawitz, and Schuurmans (1998).

\section{Modelling}

\subsection{Modelling of a canal reach}

A third order linear canal model is used in this work. This model was first used for simulation purposes by Weyer (2001). The model has been recently tested for control purposes (van Overloop et al., 2014). The model can be deduced from the SaintVenant equations using the following assumptions: (1) the advection is neglected, (2) the depth, the wet cross sectional area and the hydraulic radius are considered constant. Then the SaintVenant equations are discretized using three discretization points, then they are linearized and transformed to the Laplace domain. The result is a third order transfer function without delay, linking the upstream discharge and the downstream water level in the following form:

$G_{I R}(s)=\overbrace{\frac{1}{A_{s} S}}^{\text {Integrator }} \overbrace{\frac{\omega_{0}^{2}}{s^{2}+2 \zeta \omega_{0} s+\omega_{0}^{2}}}^{\text {Resonance }}$

Note that following this development there is no time delay in the model: the wave behaviour accounts implicitly for the time delay. Details about the model can be found in van Overloop et al. (2010b).

The integrator part has a gain that is inversely proportional to the backwater area $\left(A_{s}\right)$. The second order component is a damped oscillator with natural frequency $\omega_{0}$, damping ratio $\zeta$ and resonance peak $M_{r} . A_{s}$ is the backwater area. The natural frequency $\omega_{0}$ is approximated by the resonance frequency. For the $i$ th canal reach, the downstream water level $h_{i}(s)$ can be expressed (van Overloop et al., 2010b) as follows:

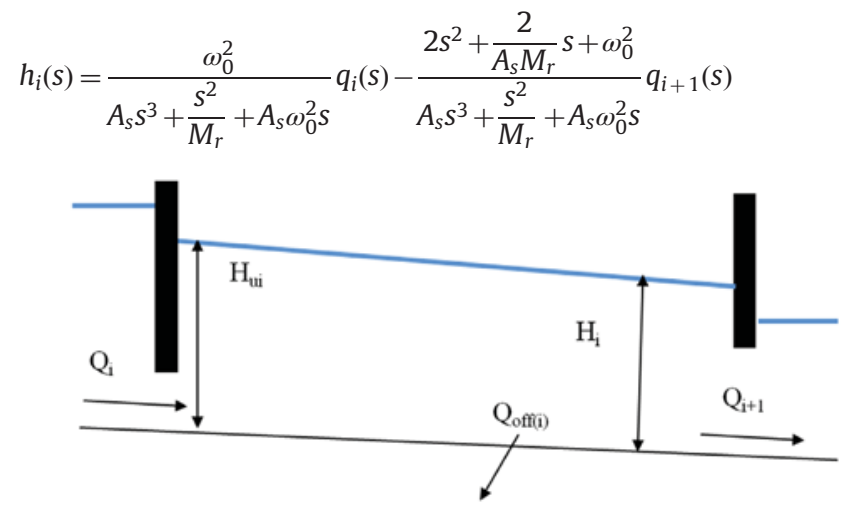


where $q_{i}$ is the upstream discharge and $q_{i+1}$ is the downstream discharge. The notation is illustrated in Fig. 2 . The quantities $h_{i}, q_{i}$ and $q_{i+1}$ are values relative to a steady state, a nominal operating point around which the non-linear system is linearized. In this way, the relative water level $h_{i}$ can be expressed as

$h_{i}(t)=H_{i}(t)-H_{0}$.

where $H_{i}(t)$ is the absolute water level and $H_{0}$ is the steady state water level.

\subsection{State space model formulation}

The objective of this work is to control the water level at the downstream end of each canal pool by changing the discharge (upstream and downstream) in the presence of known and unknown disturbances. In this case these disturbances are offtakes (discharge is taken from the canal) located at the downstream end of the canal pools.

The water level error $e_{i}$ can be written as the difference between the actual water level and the setpoint $h_{s p i}$, that is

$e_{i}(s)=h_{i}(s)-h_{s p i}$

By combining and discretizing Eqs. (2) and (4), the water level error can be expressed in terms of the input discharges. From this expression, a state space model can be constructed in the following form:

$x(k+1)=A x(k)+B u(k)+B_{d} d(k)$

where $x$ is the $n$-dimensional state vector containing the water level errors and the discharges at the previous instants, $u$ is the $m$ dimensional control vector that contains the change in gate discharge $\left(\Delta q_{i}\right), A$ is a $n \times n$ square matrix, $B$ is a $n \times m$ matrix and $B_{d}$ is a $n \times n_{b d}$ matrix, where $n_{b d}$ is the number of known disturbances, which are included in the $n_{b d}$-dimensional vector $d$. The change in discharge is expressed as

$\Delta q_{i}(k)=q_{i}(k+1)-q_{i}(k)$.

This model provides the relationship between the upstream/ downstream discharge and the downstream water level. Since the actuators are the gates, there is a need to convert the calculated discharge to gate opening. This problem is addressed in Malaterre and Baume (1999). In this work the simplest option was used: the controller calculates a control action as discharge, and then the gate openings are obtained by using the water level measurements and the inverse non-linear gate equation. If the gate opening is not physically feasible (e.g. if the gates come out of water), the maximum possible gate opening is sent to the actuator.

\section{Controller development}

\subsection{Basic predictive control, MPC-B}

The predictive control law is obtained based on Martín Sánchez and Rodellar (1996) and Camacho and Bordons (1998). The control formulation is performed in two steps: (1) a prediction of the future state vector over a prediction horizon, and (2) a minimization problem to derive the control. In the following this control method is named as MPC-B.

Prediction: To establish the prediction, a time horizon $[k, k+\lambda]$ is considered, where $k$ is the current real time instant and $\lambda$ is a time horizon to be selected as a parameter. The notation $x(k+j \mid k)$ indicates the prediction of vector $x$ for a future time instant $k+j$ within this interval. The prediction starts with the following equation:

$x(k+1 \mid k)=A x(k \mid k)+B u(k \mid k)+B_{d} d(k \mid k)$

where $x(k \mid k)=x(k), u(k \mid k)=u(k)$ and $d(k \mid k)=d(k)$.
The same prediction can be written over the entire prediction horizon $[k, k+\lambda]$

$x(k+j \mid k)=A^{j} x(k)+\sum_{n=0}^{n=j-1} A^{n} B u(k+n \mid k)+\sum_{m=0}^{m=j-1} A^{m} B_{d} d(k+m \mid k)$

for $j=1, \ldots, \lambda$.

All the single predictions can be lumped together in the following form:

$\mathbf{X}=\mathbf{A} x_{0}+\mathbf{B U}+\mathbf{B}_{\mathbf{d}} \mathbf{D}$

where $x_{0}=x(k \mid k)$.

Control: In the previous section the prediction was described over the prediction horizon, and the state equation for this horizon was expressed in the matrix form in Eq. (9). The control vector $\mathbf{U}$ is a vector containing $m \lambda$ unknowns: the values of the control vector $u$ for each reach at every time $k|k, k+1| k, \ldots, k+\lambda-1 \mid k$. The whole vector $\mathbf{U}$ is obtained through the minimization of the following cost function with constraints:

$\min _{\mathbf{U}} J_{B}=\mathbf{X}^{T} \mathbf{P X}+\mathbf{U}^{T} \mathbf{R} \mathbf{U}$

$x_{\min }<x<x_{\max }$ and $u_{\min }<u<u_{\max }$, where $\mathbf{P}(\lambda n \times \lambda n)$ and $\mathbf{R}(\lambda m \times$ $\lambda m$ ) are weighing matrices and $\mathbf{X}$ is the state vector for the whole prediction horizon. More explicitly the cost function can be written in the following form:

$J_{B}=\sum_{j=1}^{\lambda} x(k+j \mid k)^{T} P_{j} x(k+j \mid k)+\sum_{j=0}^{\lambda-1} u(k+j \mid k)^{T} R_{j} u(k+j \mid k)$

where $P_{j}$ is an $n \times n$ and $R_{j}$ is an $m \times m$ matrix.

Table 1

Steady state.

\begin{tabular}{llllllll}
\hline $\begin{array}{l}\mathbf{Q}_{\text {appr }} \\
(\mathbf{1} / \mathbf{s})\end{array}$ & $\begin{array}{l}\mathbf{S p 1} \\
(\mathbf{c m})\end{array}$ & $\begin{array}{l}\text { Sp2 } \\
(\mathbf{c m})\end{array}$ & $\begin{array}{l}\text { Sp3 } \\
(\mathbf{c m})\end{array}$ & $\begin{array}{l}\mathbf{G 1} \\
(\mathbf{c m})\end{array}$ & $\begin{array}{l}\text { G2 } \\
(\mathbf{c m})\end{array}$ & $\begin{array}{l}\text { G3 } \\
(\mathbf{c m})\end{array}$ & $\begin{array}{l}\text { W3 } \\
(\mathbf{c m})\end{array}$ \\
\hline 60 & 85 & 70 & 55 & 7.4 & 12.6 & 11.8 & 35 \\
\hline
\end{tabular}

Table 2

Setpoint change test.

\begin{tabular}{clllllllll}
\hline $\begin{array}{c}\text { Time } \\
(\mathbf{m i n})\end{array}$ & $\begin{array}{l}\mathbf{Q}_{\mathbf{a p p r}} \\
(\mathbf{1} / \mathbf{s})\end{array}$ & $\begin{array}{l}\mathbf{S p 1} \\
(\mathbf{c m})\end{array}$ & $\begin{array}{l}\mathbf{S p 2} \\
(\mathbf{c m})\end{array}$ & $\begin{array}{l}\mathbf{S p 3} \\
(\mathbf{c m})\end{array}$ & $\begin{array}{l}\mathbf{W 1} \\
(\mathbf{c m})\end{array}$ & $\begin{array}{l}\mathbf{Q}_{\mathbf{w 1}} \\
(\mathbf{1} / \mathbf{s})\end{array}$ & $\begin{array}{l}\mathbf{W 2} \\
(\mathbf{c m})\end{array}$ & $\begin{array}{l}\mathbf{Q}_{\mathbf{w 2}} \\
(\mathbf{1} / \mathbf{s})\end{array}$ & $\begin{array}{l}\mathbf{W 3} \\
(\mathbf{c m})\end{array}$ \\
\hline 0 & 60 & 85 & 70 & 55 & 90 & 0 & 90 & 0 & 35 \\
30 & 60 & 75 & 70 & 55 & 90 & 0 & 90 & 0 & 35 \\
60 & 60 & 85 & 70 & 55 & 90 & 0 & 90 & 0 & 35 \\
90 & 60 & 85 & 60 & 55 & 90 & 0 & 90 & 0 & 35 \\
180 & 60 & 85 & 70 & 55 & 90 & 0 & 90 & 0 & 35 \\
210 & 84 & 85 & 70 & 60 & 90 & 0 & 90 & 0 & 35 \\
240 & 60 & 85 & 70 & 55 & 90 & 0 & 90 & 0 & 35 \\
270 & End & & & & & & & & \\
\hline
\end{tabular}

Table 3

Disturbance test.

\begin{tabular}{rlllllllll}
\hline $\begin{array}{l}\text { Time } \\
(\mathbf{m i n})\end{array}$ & $\begin{array}{l}\mathbf{Q}_{\mathrm{appr}} \\
(\mathbf{1} / \mathbf{s})\end{array}$ & $\begin{array}{l}\mathbf{S p 1} \\
(\mathbf{c m})\end{array}$ & $\begin{array}{l}\mathbf{S p 2} \\
(\mathbf{c m})\end{array}$ & $\begin{array}{l}\mathbf{S p 3} \\
(\mathbf{c m})\end{array}$ & $\begin{array}{l}\mathbf{W 1} \\
(\mathbf{c m})\end{array}$ & $\begin{array}{l}\mathbf{Q}_{\mathbf{w 1}} \\
(\mathbf{1} / \mathbf{s})\end{array}$ & $\begin{array}{l}\mathbf{W} 2 \\
(\mathbf{c m})\end{array}$ & $\begin{array}{l}\mathbf{Q}_{\mathbf{w} 2} \\
(\mathbf{1} / \mathbf{s})\end{array}$ & $\begin{array}{l}\mathbf{W 3} \\
(\mathbf{c m})\end{array}$ \\
\hline 0 & 60 & 85 & 70 & 55 & 90 & 0 & 90 & 0 & 35 \\
30 & 60 & 85 & 70 & 55 & 75 & 20 & 90 & 0 & 35 \\
60 & 60 & 85 & 70 & 55 & 90 & 0 & 90 & 0 & 35 \\
90 & 60 & 85 & 70 & 55 & 90 & 0 & 60 & 20 & 35 \\
120 & 60 & 85 & 70 & 55 & 90 & 0 & 90 & 0 & 35 \\
150 & End & & & & & & & & \\
\hline
\end{tabular}


In this work all weighing matrices $P_{j}$ and $R_{j}$ are chosen to be equal and diagonal, $P_{j}=P$ for $j=1,2, \ldots, \lambda$ and $R_{j}=R$ for $j=0$, $1, \ldots, \lambda-1$. In other words, the weights of the optimization do not change during the prediction horizon. The matrix $R$ contains the corresponding weights on the input, the matrix $P$ contains the weights on the state.

\subsection{Controller with integral action, MPC-FB}

A controller with integral action is presented in Rodellar, Gómez, and Martín Vide (1989, 1993). Here the same controller is described using matrix formulation in order to be in similar format as MPC in Section 4.1. This controller is abbreviated as MPC-FB in the following, as it does not contain feedforward, only feedback parts. The same state as expressed in Eq. (5) is used. Then the incremental state and the incremental input can be defined in the following way:

$x_{\text {inc }}(k)=x(k)-x(k-1)$

and

$u_{\text {inc }}(k)=u(k)-u(k-1)$.

If the control action variable $u$ is chosen to be the change of discharge, $u_{i}$ is the change of change of discharge. Using the above expressions for the incremental variables, from Eq. (5) can be expressed in the following incremental form:

$x_{\text {inc }}(k+1)=A x_{\text {inc }}(k)+B u_{\text {inc }}(k)+B_{d} d_{\text {inc }}(k)$.

The control law will be calculated during a given interval, with the length of $\lambda$, called the prediction horizon: $[k, k+\lambda]$. In this formulation of predictive control constant control input is assumed over the entire prediction horizon, therefore the incremental input is zero after the first increment:

$u_{\text {inc }}(n)=0$ for $n>k$.

Using the incremental model, the state can be summed up during all the prediction horizon

$x(k+\lambda)=\left(A_{\text {sum }}+I\right) x_{0}+B_{\text {sum }} u(k)+\left[-A_{\text {sum }} x(k-1)-B_{\text {sum }} u(k-1)\right]$

where $A_{\text {sum }}$ and $B_{\text {sum }}$ are the following matrices:

$A_{\text {sum }}=\sum_{j=1}^{\lambda} A^{j} ; \quad B_{\text {sum }}=\sum_{j=1}^{\lambda} A^{j-1} B$

The sum of the state equations during the prediction horizon, Eq. (16), can be expressed in a more compact form:

$\mathbf{X}_{\mathbf{m} \mathbf{1}}=\mathbf{A}_{\mathbf{m} \mathbf{1}} x_{0}+\mathbf{B}_{\mathbf{m} \mathbf{1}} \mathbf{U}_{\mathbf{m} \mathbf{1}}+\mathbf{B}_{\mathbf{d m} \mathbf{1}} \mathbf{D}_{\mathbf{m} \mathbf{1}}$.

The terms of Eq. (18) are detailed one by one. The term $\mathbf{X}_{\mathbf{m} 1}$ is the state at the end of the prediction horizon and $\mathbf{U}_{\mathbf{m} \mathbf{1}}$ is the input vector of the system at the present instant $k$ :

$\mathbf{X}_{\mathbf{m} 1}=x(k+\lambda)$

$\mathbf{U}_{\mathbf{m} 1}=u(k)$

The matrices multiplying the state and the input are the following, respectively,

$\mathbf{A}_{\mathbf{m} \mathbf{1}}=A_{\text {sum }}+I$

$\mathbf{B}_{\mathbf{m} 1}=B_{\text {sum }}$

where the matrices $A_{\text {sum }}$ and $B_{\text {sum }}$ are defined by Eq. (17). The definitions of matrices $\mathbf{D}_{\mathbf{m} \mathbf{1}}$ and $\mathbf{B}_{\mathbf{d m} \mathbf{1}}$ are the following:

$\mathbf{B}_{\mathbf{d m} 1}=\left[-A_{\text {sum }} x(k-1)-B_{\text {sum }} u(k-1)\right]$

Table 4

Test 1.

\begin{tabular}{|c|c|c|c|c|c|c|}
\hline Pool & $\begin{array}{l}\text { Offtake initial } \\
\text { flows }\left(\mathbf{m}^{3 / s}\right)\end{array}$ & $\begin{array}{l}\text { Check initial flows } \\
\left(\mathbf{m}^{3 / s}\right)\end{array}$ & $\begin{array}{l}\text { Scheduled offtake changes at } \\
2 \mathrm{~h}\left(\mathrm{~m}^{3} / \mathrm{s}\right)\end{array}$ & $\begin{array}{l}\text { Resulting check } \\
\text { flows }\left(\mathbf{m}^{3} / \mathbf{s}\right)\end{array}$ & $\begin{array}{l}\text { Unscheduled offtake changes at } \\
14 \mathrm{~h}\left(\mathrm{~m}^{3} / \mathrm{s}\right)\end{array}$ & $\begin{array}{l}\text { Resulting check } \\
\text { flows }\left(\mathbf{m}^{3} / \mathbf{s}\right)\end{array}$ \\
\hline Heading & - & 11.0 & - & 13.5 & - & 11.5 \\
\hline 1 & 1.0 & 10.0 & - & 12.5 & - & 10.5 \\
\hline 2 & 1.0 & 9.0 & - & 11.5 & - & 9.5 \\
\hline 3 & 1.0 & 8.0 & - & 10.5 & - & 8.5 \\
\hline 4 & 1.0 & 7.0 & - & 9.5 & - & 7.5 \\
\hline 5 & 1.0 & 6.0 & 1.5 & 7.0 & - & 5.0 \\
\hline 6 & 1.0 & 5.0 & 1.0 & 5.0 & -2.0 & 5.0 \\
\hline 7 & 1.0 & 4.0 & - & 4.0 & - & 4.0 \\
\hline 8 & 1.0 & 3.0 & - & 3.0 & - & 3.0 \\
\hline
\end{tabular}

a

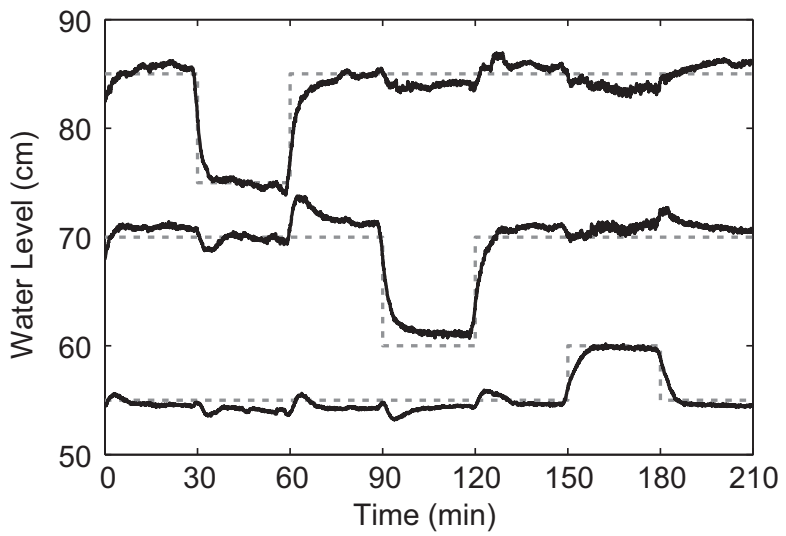

b

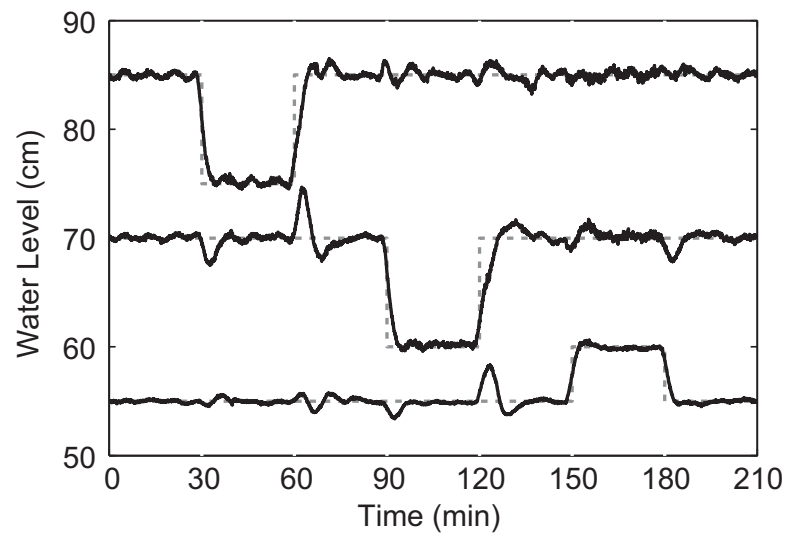

Fig. 3. Known setpoint change, Test 1 , with continuous line the measured water levels in the three pools, with horizontal dashed line the setpoint and with vertical dashed line** the time when the disturbance occurred is shown: (a) MPC-B and (b) MPC-OF. 
a

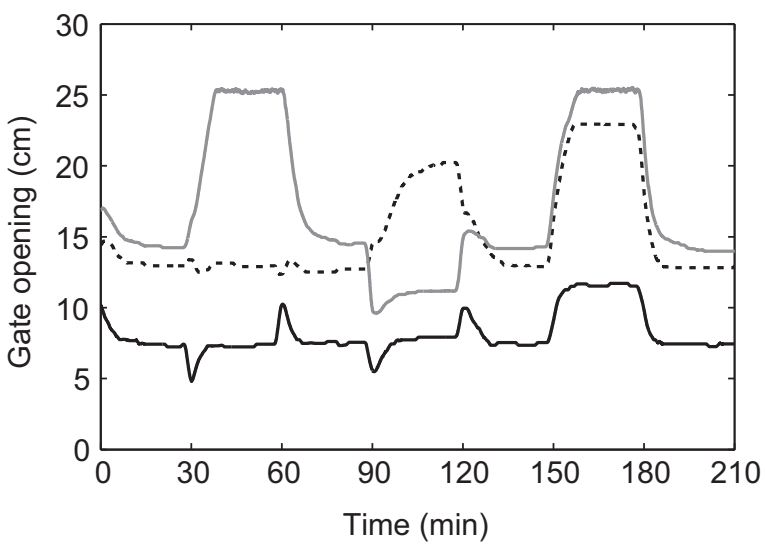

b

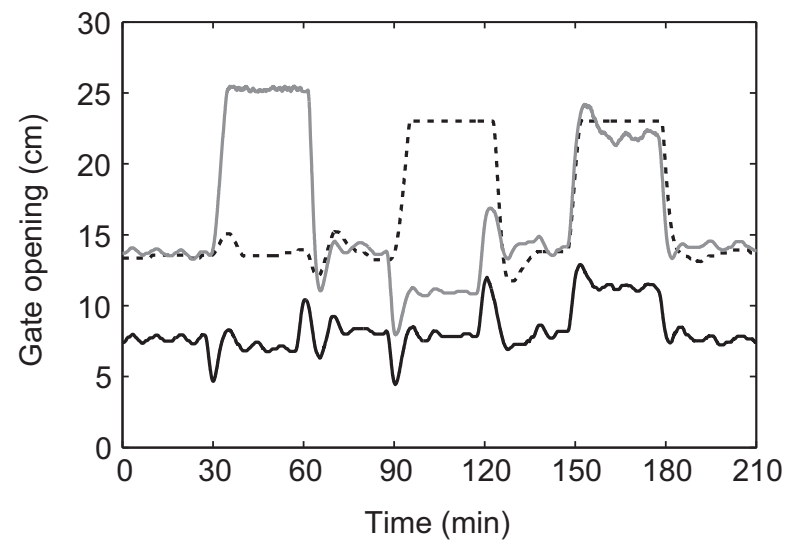

Fig. 4. Gate openings, known setpoint change, Test 1, Gate 1: black solid line, Gate 2: gray line, Gate 3: black dashed line: (a) MPC-B and (b) MPC-OF.

$\mathbf{D}_{\mathrm{m} 1}=1$

A predictive control objective associated to Eq. (18) could be to find the control vector $\mathbf{U}_{\mathbf{m} 1}$ such that the state vector at $k+\lambda$, $x(k+\lambda \mid k)=\mathbf{X}_{\mathbf{m} 1}$ is as close as possible to the setpoint through the minimization of

$\min _{\mathbf{U}_{\mathbf{m} \mathbf{1}}} J_{F B}=x(k+\lambda \mid k)^{T} \mathbf{P}_{\mathbf{m} \mathbf{1}} x(k+\lambda \mid k)+u(k \mid k)^{T} \mathbf{R}_{\mathbf{m} \mathbf{1}} u(k \mid k)$,

in the matrix form

$\min _{\mathbf{U}_{\mathbf{m} \mathbf{1}}} J_{F B}=\mathbf{X}_{\mathbf{m} \mathbf{1}}^{T} \mathbf{P}_{\mathbf{m} \mathbf{1}} \mathbf{X}_{\mathbf{m} \mathbf{1}}+\mathbf{U}_{\mathbf{m} \mathbf{1}}^{T} \mathbf{R}_{\mathbf{m} \mathbf{1}} \mathbf{U}_{\mathbf{m} \mathbf{1}}$.

where $\mathbf{P}_{\mathbf{m} \mathbf{1}}(n \times n)$ and $\mathbf{R}_{\mathbf{m} \mathbf{1}}(m \times m)$ are weighing matrices. $\mathbf{P}_{\mathbf{m} \mathbf{1}}$ is a diagonal matrix with size $n \times n$ that has zero entries for the states that are not to be penalized and non-zero entries $\left(p_{m 1}\right)$ for the states that are to be penalized. In this work these states are the water level errors.

\subsection{Offset-free predictive control, MPC-OF}

The controller described in this section is denoted as MPC-OF in the following. In this step the controller objectives presented in Section 4.1 (Eq. (10)) with the one presented above in Section 4.2 (Eq. (25)) are combined. The aim is to combine the advantages of both strategies. The basic method (MPC-B) has good reaction to known disturbances, but it is not able to respond without offset to unknown disturbances. The simplified method (MPC-FB) reaches always offset free response, however, its response to known disturbances is not satisfactory. The control objective defined here aims to find the control vector $\mathbf{U}$ (including $u(k \mid k) \ldots u(k+\lambda-1 \mid k)$ ), to balance between the anticipatory action $\left(J_{B}\right.$, Eq. (10)) and the integral action $\left(J_{F B}\right.$, Eq. (25)) of the MPC. MPC-B, described in Section 4.1, incorporates the known future disturbances into its objective function. On the other hand, MPC-FB (Section 4.2) has integral action and the offset free property. In order to keep the goal of both controllers, the balance between the two actions is pursued by combining these two objective functions:

$J=J_{B}+J_{F B}$

This performance criterion has two parts, one belonging to each formulation. It can be written in a more compact form as

$\min _{\mathbf{U}} J=\mathbf{X}_{\mathbf{m} 4}^{T} \mathbf{P}_{\mathbf{m} \mathbf{4}} \mathbf{X}_{\mathbf{m} \mathbf{4}}+\mathbf{U}^{T} \mathbf{R U}$.

where

$\mathbf{X}_{\mathbf{m} 4}=\mathbf{A}_{\mathbf{m} 4} \chi_{0}+\mathbf{B}_{\mathbf{m} 4} \mathbf{U}+\mathbf{B}_{\mathbf{d m} 4} \mathbf{D}_{\mathbf{m} 4}$ and the terms are explained one by one. The state vector $\mathbf{X}_{\mathbf{m} \mathbf{4}}$ is compiled in the following form:

$\mathbf{X}_{\mathrm{m} 4}=\left[\begin{array}{c}\mathbf{X} \\ \mathbf{X}_{\mathrm{m} 1}\end{array}\right]$

where $\mathbf{x}$ contains the state all the time instants during the prediction horizon and $\mathbf{X}_{\mathbf{m} \mathbf{1}}=x(k+\lambda)$.

The vectors $x_{0}$ and $\mathbf{U}$ are the same as in Eq. (9). The input vector $\mathbf{U}$ contains the input during all the prediction horizon. This is the same input vector used in the previously developed MPC, in Section 4.1. However, the input vector used in this section $\mathbf{U}_{\mathbf{m} 1}$ contains the input only for the present instant. Therefore the combination of the two state spaces is based on connecting both vectors as follows: the first part (of length $m$ ) of the input vector $\mathbf{U}$ (that is $u(k)$ ) will be made equal to the input vector $\mathbf{U}_{\mathbf{m} 1}$, as

$\mathbf{U}_{\mathbf{m} 1}=\mathbf{U}(1: m)$.

The matrices are also combined using the corresponding matrices from the two formulations:

$A_{m 4}=\left[\begin{array}{c}A \\ A_{m 1}\end{array}\right]$

$\mathbf{B}_{\mathbf{m} 4}=\left[\begin{array}{cc}\mathbf{B} & \\ \mathbf{B}_{m 1} & 0_{n \times m(\lambda-1)}\end{array}\right]$.

Matrix $\mathbf{B}_{\mathbf{m} 4}$ has some zero entries. The reason for this is the different lengths of the input vectors: while the input vector $\mathbf{U}$ of the MPC from Eq. (9) is $m \lambda$ long, the input vector of the method presented here has an input vector $\mathbf{U}_{\mathbf{m} 1}$ with length of $m$.

The disturbance vector $\mathbf{D}_{\mathbf{m} \mathbf{4}}$ is the combination of the disturbance vector of the two methods:

$\mathbf{D}_{\mathrm{m} 4}=\left[\begin{array}{c}\mathbf{D} \\ \mathbf{D}_{\mathrm{m} 1}\end{array}\right]$.

Just as the disturbance vector, the matrix multiplying the disturbance vector is the combination of the two matrices from the two methods:

$B_{\mathrm{dm} 4}=\left[\begin{array}{ll}B_{\mathrm{d}} & \left.\mathbf{B}_{\mathrm{dm} 1}\right]\end{array}\right.$.

The weighing matrix $\mathbf{P}_{\mathbf{m} 4}$ has the following form:

$\mathbf{P}_{\mathbf{m} \mathbf{4}}=\left[\begin{array}{cc}\mathbf{P} & 0_{n \lambda \times n} \\ 0_{n \times n \lambda} & \mathbf{P}_{\mathbf{m} \mathbf{1}}\end{array}\right]$. 

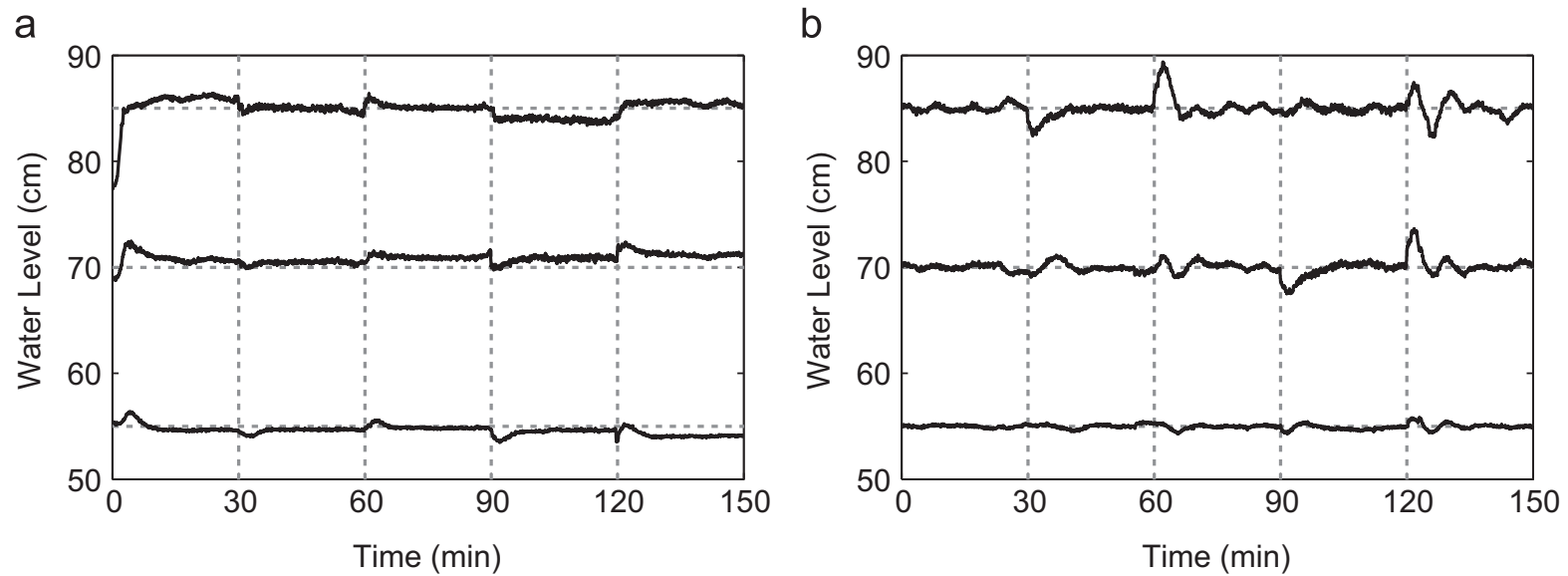

Fig. 5. Known disturbance, Test 2, with continuous line the measured water levels in the three pools, with horizontal dashed line the setpoint and with vertical dashed line the time when the disturbance occurred is shown: (a) MPC-B and (b) MPC-OF.
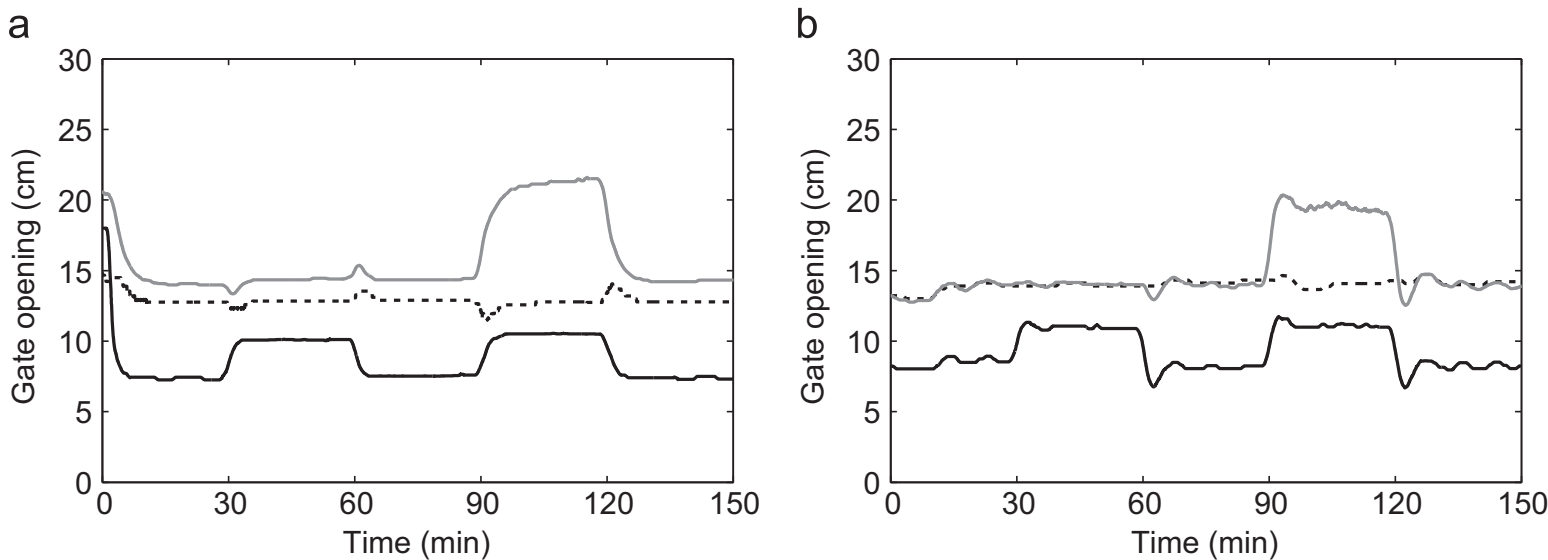

Fig. 6. Gate opening, known disturbance, Test 2, Test 1, Gate 1: black solid line, Gate 2: gray line, Gate 3: black dashed line: (a) MPC-B and (b) MPC-OF.

a

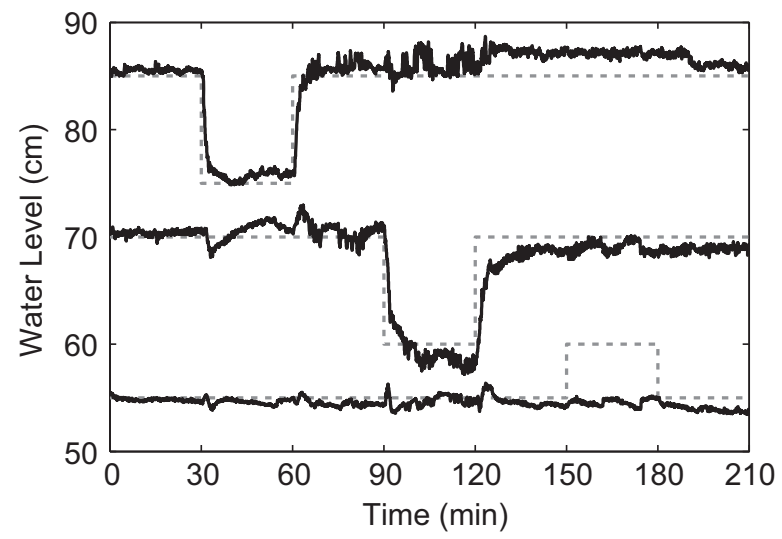

b

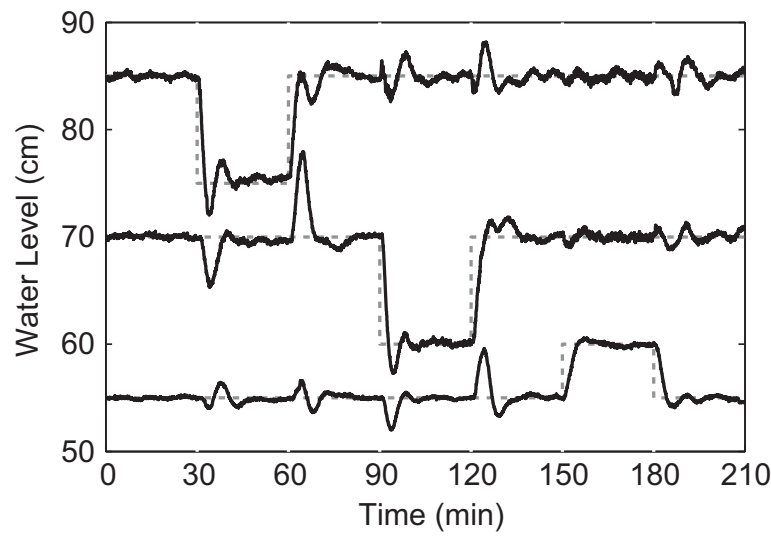

Fig. 7. Setpoint change unknown in advance, Test 3, with continuous line the measured water levels in the three pools, with dashed line the setpoint: (a) MPC-B and (b) MPC-OF.

This matrix is the combination of the weighing matrix $\mathbf{P}$ presented in Section 4.1 under Eq. (10) and the matrix $\mathbf{P}_{\mathbf{m} 1}$ from Section 4.2, in Eq. (26).

The weighing matrix $\mathbf{R}$ is the same presented in Section 4.1 after Eq. (10). Finally, the control vector $\mathbf{U}$ is obtained through the solution of the problem defined by Eqs. (28) and (29) at each sampling instant $k$. The tuning is detailed in Section 5.5.

\subsection{Model predictive control with integral action, MPC-I}

Model predictive control with integral action based on Wang (2009) is implemented. This controller is noted as MPC-I in the following. In this formulation, a new state space vector $x_{\text {int }}$ is constructed by combining the incremental variable (Eq. (12)) and the output: $x_{\text {int }}=\left[x_{i}(k) y(k)\right]^{T}$. 
a

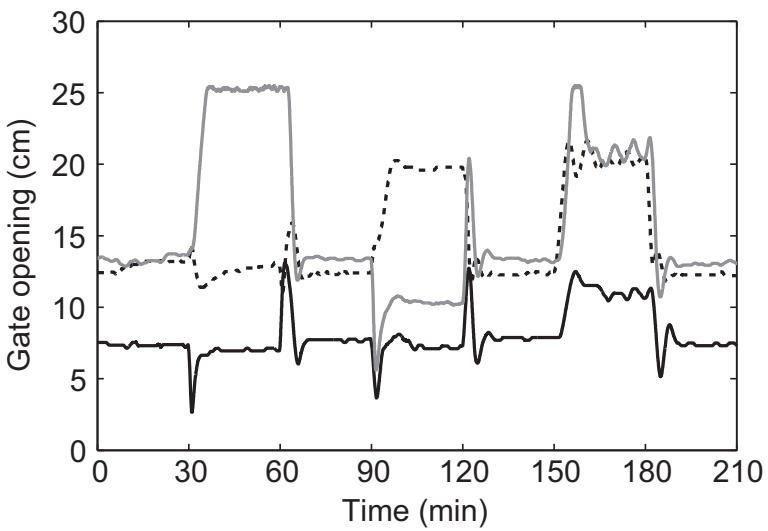

b

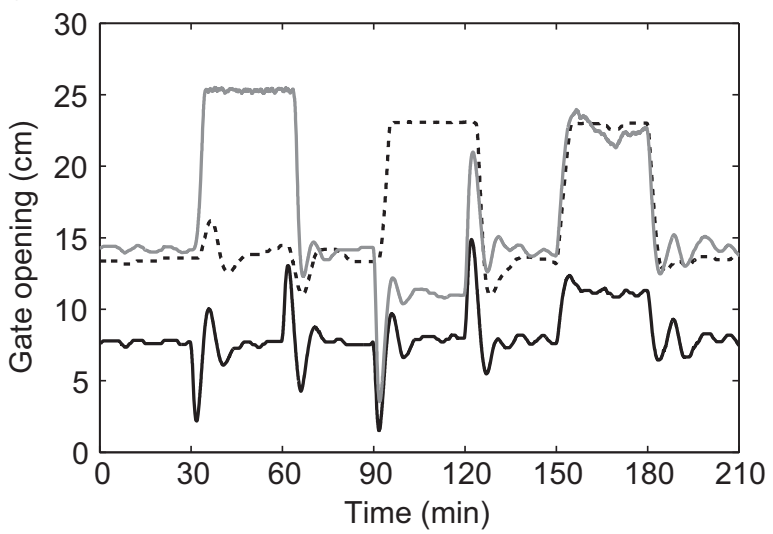

Fig. 8. Gate opening, setpoint change unknown in advance, Test 3, Gate 1: black solid line, Gate 2: gray line, Gate 3: black dashed line: (a) MPC-B and (b) MPC-OF.

a

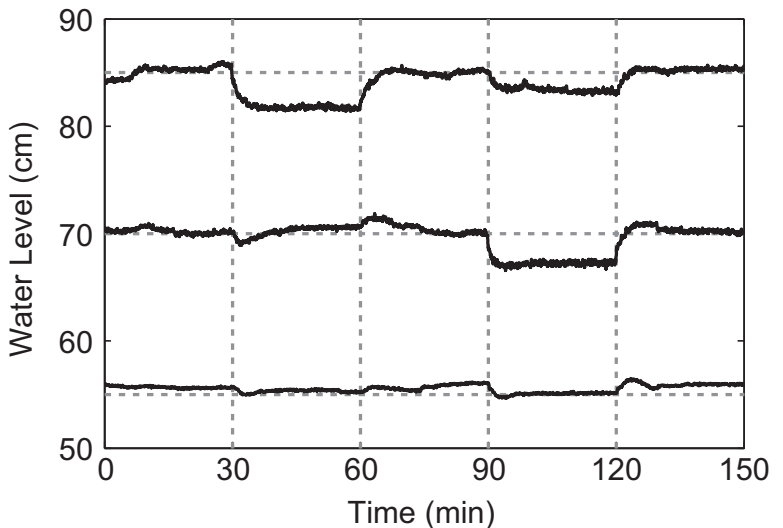

b

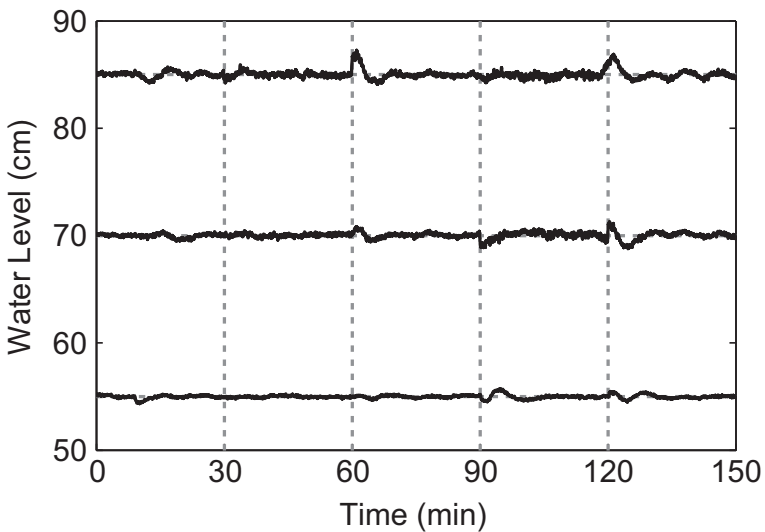

Fig. 9. Unknown disturbance, Test 4, with continuous line the measured water levels in the three pools, with horizontal dashed line the setpoint and with vertical dashed line the time when the disturbance occurred is shown: (a) MPC-B and (b) MPC-OF.
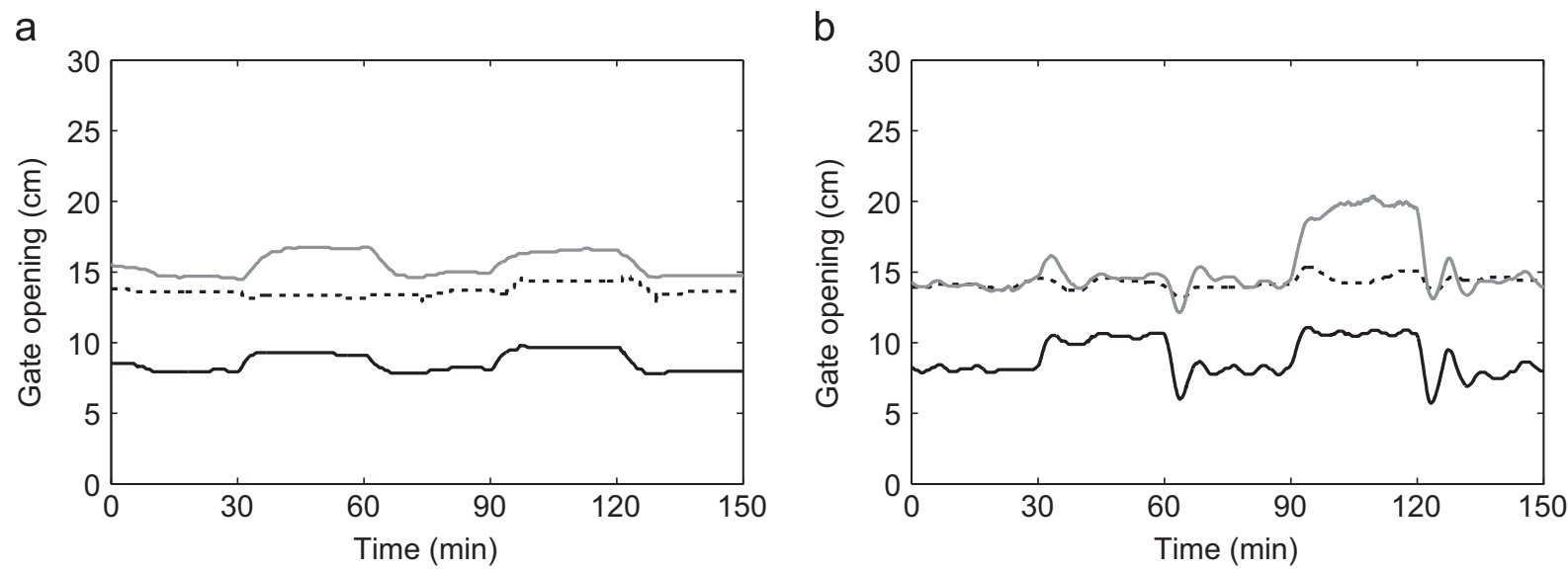

Fig. 10. Gate opening, unknown disturbance, Test 4, Gate 1: black solid line, Gate 2: gray line, Gate 3: black dashed line: (a) MPC-B and (b) MPC-OF.

Using this state vector an augmented state space can be constructed

$\left[\begin{array}{c}x_{i}(k+1) \\ y(k+1)\end{array}\right]=\left[\begin{array}{cc}A & o_{m}^{T} \\ C A & 1\end{array}\right]\left[\begin{array}{c}x_{i}(k) \\ y(k)\end{array}\right]+\left[\begin{array}{c}B \\ C B\end{array}\right] u_{i}(k)$

$y(k)=\left[\begin{array}{ll}o_{m} & 1\end{array}\right]\left[\begin{array}{c}x_{i}(k) \\ y(k)\end{array}\right]$

where $o_{m}$ is a horizontal vector of zeros with length equal to the original state. Then MPC can be developed as introduced in Section 4.1 using the augmented state vector $x_{\text {int }}$.

\subsection{Model predictive control with observer, MPC-O}

Model predictive control with observer based on Pannocchia \& Rawlings (2003) is implemented. It is denoted as MPC-O in the following.

Using state and output disturbances and augmenting the system with an observer, the following expression can be written:

$$
\left[\begin{array}{c}
\hat{x}_{k+1 \mid k} \\
\hat{d}_{k+1 \mid k}
\end{array}\right]=\left[\begin{array}{cc}
A & B_{d} \\
0 & I
\end{array}\right]\left[\begin{array}{l}
\hat{x}_{k \mid k-1} \\
\hat{d}_{k \mid k-1}
\end{array}\right]+\left[\begin{array}{l}
B \\
0
\end{array}\right] u_{k}
$$




$$
+\left[\begin{array}{l}
L_{1} \\
L_{2}
\end{array}\right]\left(y_{k}-C \hat{x}_{k \mid k-1}-C_{d} \hat{d}_{k \mid k-1}\right)
$$

where $\hat{x}$ is the observer state, $\hat{d}$ is the observed disturbance, $L_{1}$ and $L_{2}$ are the gains of the observer and $B_{d}, C_{d}$ are matrices belonging to the disturbance model constructed using the following conditions: (1) in order to create an observer for the state space $(A, C)$ should be observable; and (2) according to Pannocchia \& Rawlings (2003), in order to achieve offset-free control the number of disturbance states should be equal to the number of measurements. Any $B_{d}$ and $C_{d}$ can be chosen arbitrarily as long as the following condition is kept:

$\operatorname{rank}\left[\begin{array}{cc}I-A & -B_{d} \\ C & C_{d}\end{array}\right]=n+n_{d}$

where $n$ is the number of states and $n_{d}$ is the number of disturbance states.

\section{Test cases for the control algorithms}

Several scenarios are established in order to test the predictive controllers during this work. Numerical simulations have been carried out by the SIC software (Simulation and integration of control for canals) (Malaterre, 2012) that solves numerically using finite differences the Saint-Venant equations. For the physical tests in the experimental canal UPC-PAC, all the algorithms have been implemented using Matlab (Mathworks, 2008).

All tests start and finish with the steady state conditions as shown in Table 1 . The discharge $\left(Q_{\text {appr }}\right)$ is approximately $60 \mathrm{l} / \mathrm{s}$. The setpoint of the water level in the first pool (Sp1) is $85 \mathrm{~cm}$, in the second pool (Sp2) is $70 \mathrm{~cm}$ and in the third pool (Sp3) is $55 \mathrm{~cm}$. The gate openings to achieve the given water levels in each pool are shown for the three gates (G1, G2 and G3). The height of the final weir (W3) was set to $35 \mathrm{~cm}$ (See Fig. 2).

\subsection{Test 1: setpoint changes}

This test contains three consecutive setpoint changes. First at Pool 1, then after reaching the original situation, a setpoint change is required in Pool 2 and finally in Pool 3. All setpoint changes are $10 \mathrm{~cm}$ (except for the change in Pool 3), this value being more than $10 \%$ of the actual water level. Since the last pool has a weir at the downstream end, the last setpoint change implies change in the discharge. Table 2 shows the test step by step. The columns $S p$ show the actual setpoints, and the columns $W$ and $Q_{w}$ show the weir height and the approximate weir discharge, respectively. In this test all offtakes are closed, the weir height being at its maximum $(90 \mathrm{~cm})$. There is flow only over Weir 4 , which leaves the canal.

\subsection{Test 2: reaction to disturbances}

The disturbance rejection was tested by using the lateral weirs (See Table 3). In this test two disturbances occur: after $30 \mathrm{~min}$, the Weir 1 at the downstream end of Pool 1 is opened and it is closed after $60 \mathrm{~min}$. At $90 \mathrm{~min}$ the weir at the end of the second reach (Weir 2) is opened for $30 \mathrm{~min}$. The offtake is closed at $120 \mathrm{~min}$ and the test is finished at $150 \mathrm{~min}$. In both cases, the offtake discharge is about $20 \mathrm{l} / \mathrm{s}$. This is one-third of the actual discharge, hence it is a considerable change.

\subsection{Test 3 and Test 4}

The setpoint changes in Test 3 are the same as in Test 1, but their timing and magnitude are unknown by the controller in advance. The same is true for the disturbance test: Test 4 is similar to Test 2, but with disturbances that are unknown all the time for the controller.

\subsection{Test on the ASCE Test Canal 2}

The controllers were also evaluated using the ASCE test cases. The test scenario is $24 \mathrm{~h}$ long and detailed in Table 4 . It is Test 1 from the described benchmark tests (Clemmens et al., 1998). The first $12 \mathrm{~h}$ of the test contain known changes and the second part of the test, from 12 to $24 \mathrm{~h}$, contains unknown changes.

As this is a longer canal and the second order waves are not so significant, this canal was modeled for control purposes using the Integrator Delay model (Schuurmans, Bosgra, \& Brouwer, 1995).

\subsection{Tuning parameters}

The general tuning parameters $\left(p_{i}, r_{i}, \lambda\right)$ were the same as chosen previously for controller tuning, while the tuning parameter of the presented offset-free method has been chosen in the following way: the weight in the integration procedure was increased until offset-free control was achieved in the given case. As a general guidance this value can be chosen using Bryson's rule (Bryson, 1975): the weights are the reciprocals of the squares of the maximum allowed values:

$$
p_{i}=\frac{1}{e M A V E^{2}}
$$

where eMAVE is the Maximum Allowed Value Estimate of the water level error, and $p_{i}$ is a diagonal entry of the weighing matrix $P_{j}$ from Eq. (11). The matrices $P_{j}(j=1, . . \lambda)$ are diagonal matrices, having $p_{i}$ entry for the error states and zero entry for the other states. The matrix $R_{j}(j=1, . . \lambda-1)$ is also a diagonal matrix, with $r_{i}$ at all diagonal entries. The matrix $P_{m 1}$ has similar structure as $P_{j}$ : all entries are zero except the diagonal entries corresponding to the error states. There the entries are $p_{m 1}$. The final tuning values of the offset free test are $p_{i}=1111, r_{i}=12,346$ for the disturbance and $r_{i}=4444$ for the setpoint test. For both tests, we have $p_{m 1}=1111$.

\section{Results}

\subsection{Experimental results}

The offset-free method (MPC-OF) was implemented and the above described four tests were carried out in the experimental facility of the Technical University of Catalonia described in Section 2.1. The results section shows the measured water level signals obtained from the SCADA system of the UPC-PAC. The prediction horizon was set to 15 samples $(\lambda=15)$ for the experimental tests. A sampling period of $10 \mathrm{~s}$ was used for measurements and control, so that the gates were adjusted at every $10 \mathrm{~s}$.

The first case is the known setpoint change (Test 1), without (MPC-B, Figs. 3a and 4a) and with offset free method (MPC-OF, Figs. $3 \mathrm{~b}$ and $4 \mathrm{~b}$ ). Both controllers were able to follow the desired setpoint changes. In the UPC-PAC the minimum movement for the gate to change its position is restricted. This causes different effects with the different methods: for the controller without offset free method the water level settles some millimeters above or under the setpoint, for example, in Fig. 3a the water level in Pool 2 between 0 and $30 \mathrm{~min}$ was slightly over the setpoint (shown with gray dashed line). The reason is that the controller would make a slight movement to correct the gate position, but the gate cannot make such small movement, hence it stays over setpoint. In case of the offset free method (MPC-OF, Figs. 3b and 
Table 5

UPC-PAC, known changes.

Table 5 modified

\begin{tabular}{|c|c|c|c|c|c|c|c|c|c|c|c|c|}
\hline \multirow[t]{3}{*}{ Name } & \multicolumn{4}{|c|}{ MAE (\%) } & \multicolumn{4}{|c|}{ IAE (\%) } & \multicolumn{4}{|c|}{ StE (\%) } \\
\hline & \multicolumn{2}{|c|}{ Setpoint } & \multicolumn{2}{|c|}{ Disturbance } & \multicolumn{2}{|c|}{ Setpoint } & \multicolumn{2}{|c|}{ Disturbance } & \multicolumn{2}{|c|}{ Setpoint } & \multicolumn{2}{|c|}{ Disturbance } \\
\hline & Max. & Avg. & Max. & Avg. & Max. & Avg. & Max. & Avg. & Max. & Avg. & Max. & Avg. \\
\hline MPC & 11.7 & 7.7 & 1.6 & 0.9 & 0.5 & 0.4 & 0.1 & 0.1 & 0.8 & 0.6 & 0.1 & 0.1 \\
\hline MPC-I & 18.0 & 14.9 & 5.2 & 4.7 & 7.6 & 5.6 & 1.7 & 1.4 & 5.2 & 3.4 & 1.5 & 0.9 \\
\hline MPC-OF & 12.4 & 8.3 & 1.4 & 0.9 & 1.2 & 0.7 & 0.1 & 0.0 & 0.7 & 0.5 & 0.1 & 0.1 \\
\hline
\end{tabular}

Table 6

UPC-PAC, unknown changes.

Table 6 modified

\begin{tabular}{|c|c|c|c|c|c|c|c|c|c|c|c|c|}
\hline \multirow[t]{3}{*}{ Name } & \multicolumn{4}{|c|}{ MAE (\%) } & \multicolumn{4}{|c|}{ IAE (\%) } & \multicolumn{4}{|c|}{ StE (\%) } \\
\hline & \multicolumn{2}{|c|}{ Setpoint } & \multicolumn{2}{|c|}{ Disturbance } & \multicolumn{2}{|c|}{ Setpoint } & \multicolumn{2}{|c|}{ Disturbance } & \multicolumn{2}{|c|}{ Setpoint } & \multicolumn{2}{|c|}{ Disturbance } \\
\hline & Max. & Avg. & Max. & Avg. & Max. & Avg. & Max. & Avg. & Max. & Avg. & Max. & Avg. \\
\hline $\mathrm{MPCl}$ & 15.5 & 11.9 & 4.6 & 3.4 & 1.7 & 1.0 & 1.1 & 0.8 & 1.2 & 0.5 & 1.3 & 0.9 \\
\hline MPC-I & 16.2 & 12.0 & 4.5 & 4.1 & 3.1 & 2.9 & 1.3 & 0.9 & 1.9 & 1.2 & 0.6 & 0.4 \\
\hline MPC-OF & 16.6 & 12.8 & 3.8 & 2.6 & 1.4 & 0.9 & 0.4 & 0.3 & 0.2 & 0.1 & 0.1 & 0.1 \\
\hline MPC-FB & 16.6 & 13.0 & 4.0 & 2.8 & 1.4 & 0.9 & 0.4 & 0.2 & 0.2 & 0.1 & 0.1 & 0.0 \\
\hline
\end{tabular}

a

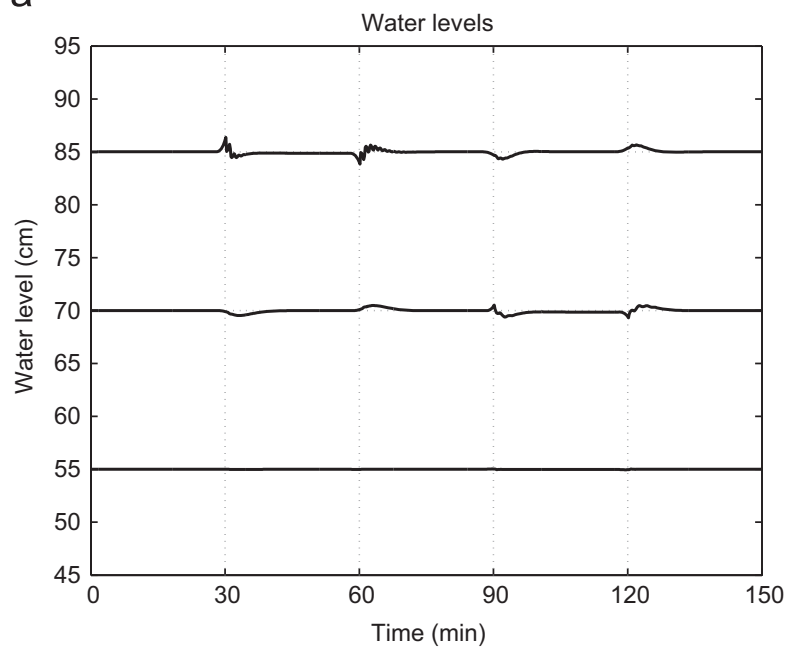

b

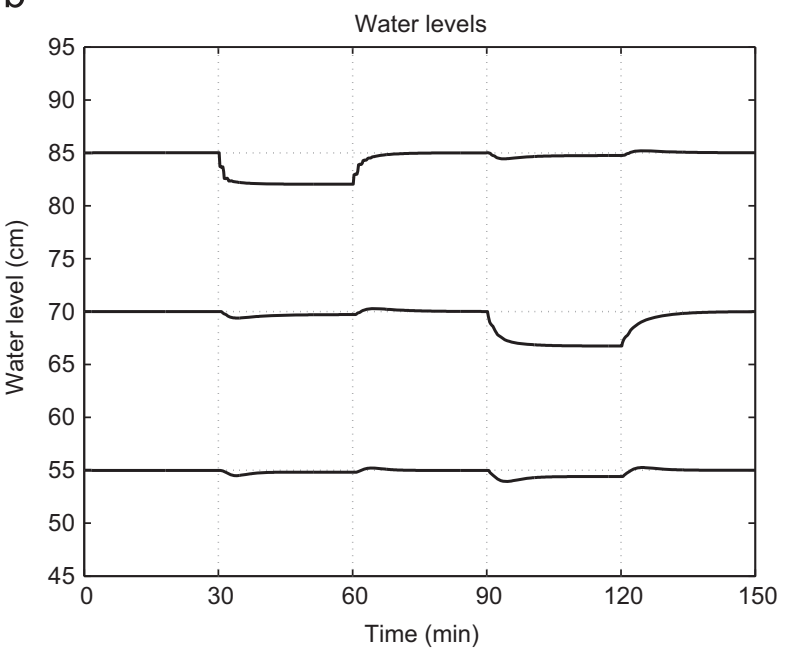

Fig. 11. Disturbance test, MPC-B: (a) known and (b) unknown.

a

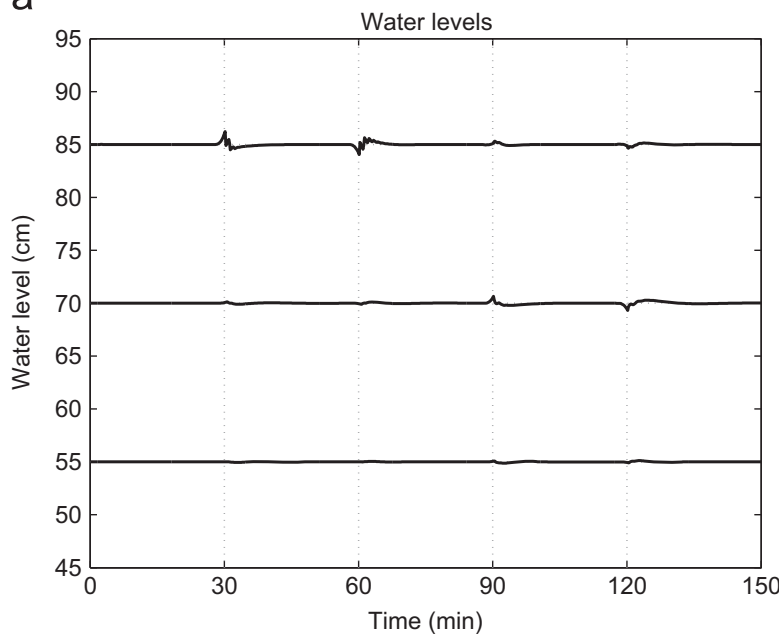

b

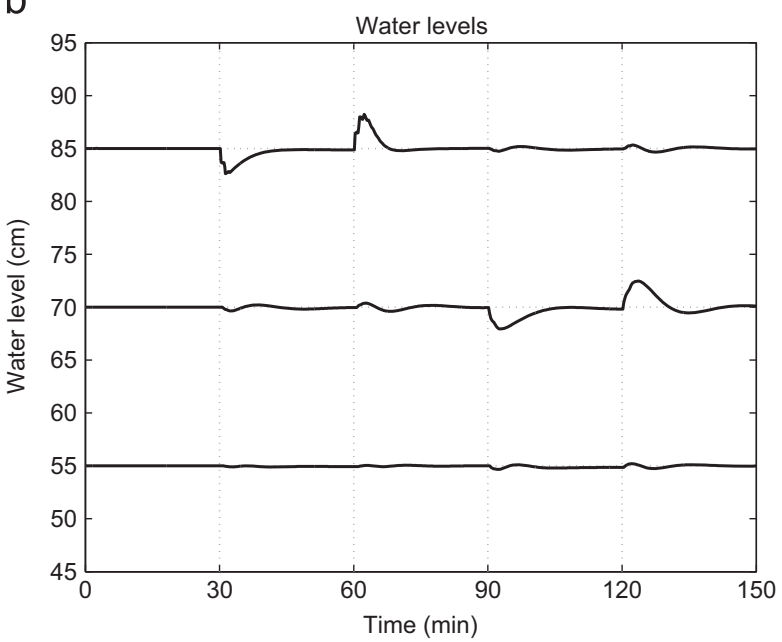

Fig. 12. Disturbance test, MPC-OF: (a) known and (b) unknown. 
a

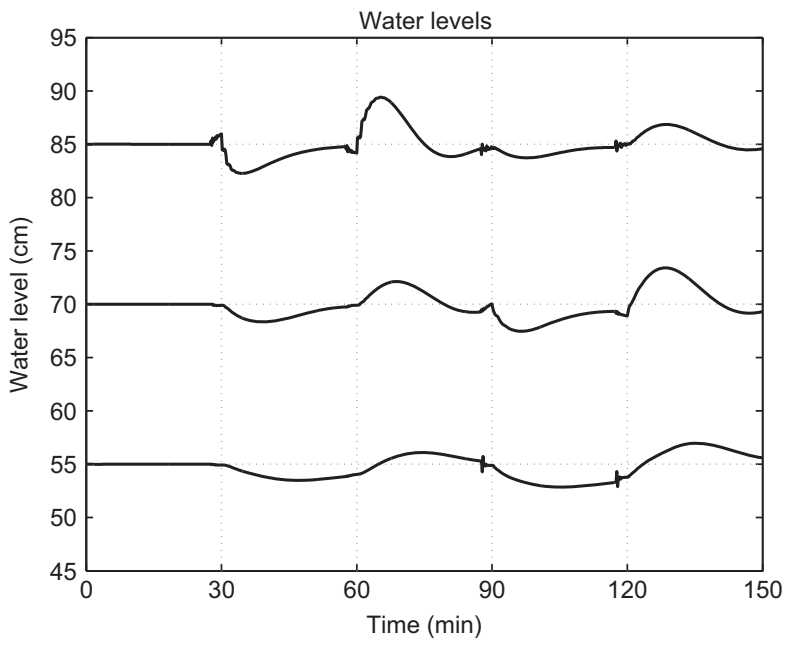

b

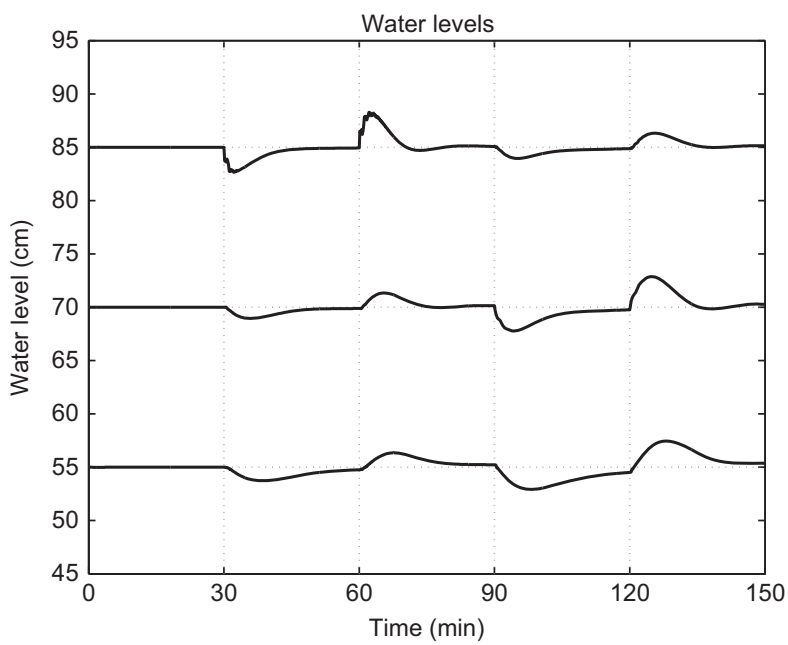

Fig. 13. Disturbance test, MPC-I: (a) known and (b) unknown.

4b) the water levels are slightly oscillating around setpoint. The reason is similar, when the water level is for example above the setpoint the controller calculates a small movement to close the gate. However, this does not occur, due to the minimum gate movement constraint. Then the controller calculates bigger and bigger movements until the movement would be physically realizable. However, this big movement would cause an offset in the other direction and, following the mentioned example, the water level would be under the setpoint and the whole process of corrections would start again. This phenomenon causes the small oscillations in the water level and in the gate movements. It can be avoided with a simple constraint in the gate movement, but it would also result in a small offset just like in the first case. The different behaviour of the controllers can also be seen at $60 \mathrm{~min}$, when the setpoint in Pool 1 is changed back to the original. MPCOF makes a sharp increase in the water level of Pool 2, and then it goes back fast to the setpoint, while MPC-B has smaller overshoot, but it approaches slowly the setpoint and does not reach it before $90 \mathrm{~min}$. Note that in both cases with (MPC-OF, Figs. 3b and 4b) and without (MPC-B, Figs. 3a and 4a) the offset free method, the result of the control action is observed before the desired setpoint change, since the setpoint change is known beforehand and the controller is able to act in advance.

Similar conclusions can be drawn from the comparison of the known disturbance case (Test 2) without (MPC-B, Figs. 5a and 6a) and with offset-free method (MPC-OF, Figs. 5b and 6b). Both controllers were able to keep the water levels at setpoint while known disturbances occurred.

However, in case of unknown changes, the performance of the two controllers is different. The controller without offset free method is not able to carry out the unknown setpoint change test (MPC-B, Figs. 7a and 8a): for Pool 3, between 150 and 180 min the setpoint changes from $55 \mathrm{~cm}$ to $60 \mathrm{~cm}$ and the controller is not able to follow this change, the water level remaining at $55 \mathrm{~cm}$. Note that this problem occurs only in case of the setpoint change for Pool 3. The reason is that in order to change the water level of Pool 3 the discharge should be changed, since there is a weir at the downstream end of Pool 3 that determines the discharge-water level relationship. However, the predictive model in MPC should know the outflow from the canal, which in this case changes at every time step as the water level reaches the new setpoint. The discharge change can also be seen from the gate movements (Fig. 8a and b): in order to increase the level in Pool 3 between 150 and $180 \mathrm{~min}$ more discharge is needed in to the canal, therefore

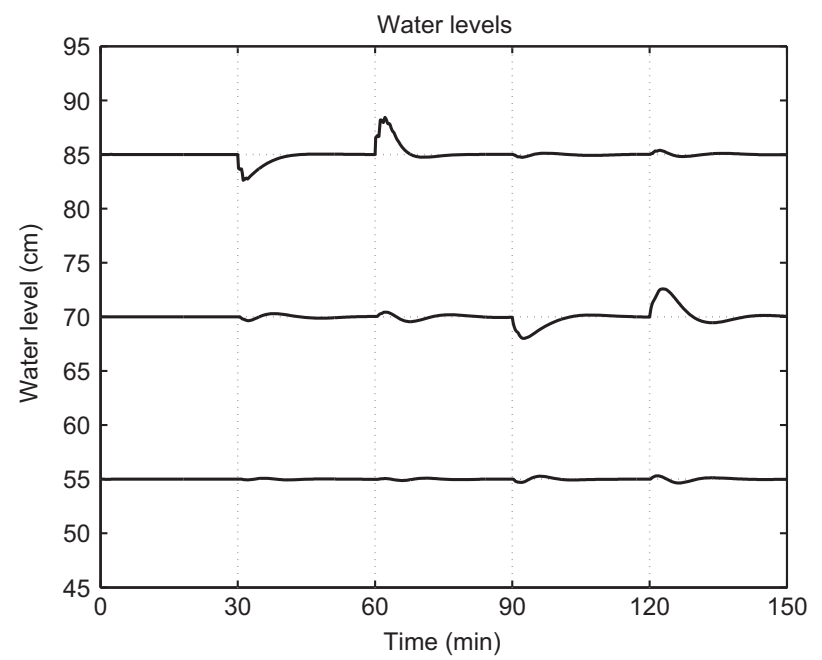

Fig. 14. MPC-FB: (a) disturbance.

Gate 1 should be more open, as it is in case of the MPC-OF (Fig. 8b). The controller with offset free method (MPC-OF, Figs. 7b and $8 b$ ) is able to tackle the problem: all the three setpoints are kept during the test.

The controller without offset free method also failed during the unknown disturbance test (MPC-B, Figs. 9a and 10a): when the disturbance occurred in Pool 1 between $30 \mathrm{~min}$ and $60 \mathrm{~min}$, the water level was not kept at setpoint. Also when the disturbance occurred in Pool 2 between 90 min and 120 min neither the water level in Pool 1 nor in Pool 2 was kept at the setpoint. These changes were difficult for the controller because they involved unknown change in discharge. However, the offset-free method solved the problem again (MPC-OF, Figs. 9b and 10b): all the three setpoints were kept during all the experiment in the three pools.

\subsection{Numerical results on the UPC-PAC}

The MPC-B, MPC-FB, MPC-OF and MPC-I are implemented numerically. In order to use MPC-O, the state space model should be observable (Section 4.5). In the current state space formulation the system was not observable, hence the MPC-O was not implemented. This might be due to the structure of the model, and it might be possible to overcome by restructuring the model 
a

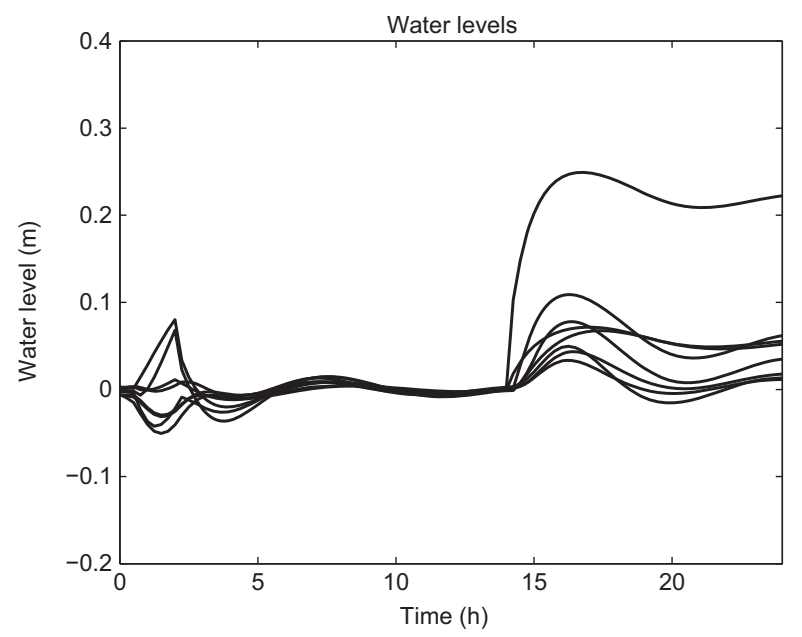

b

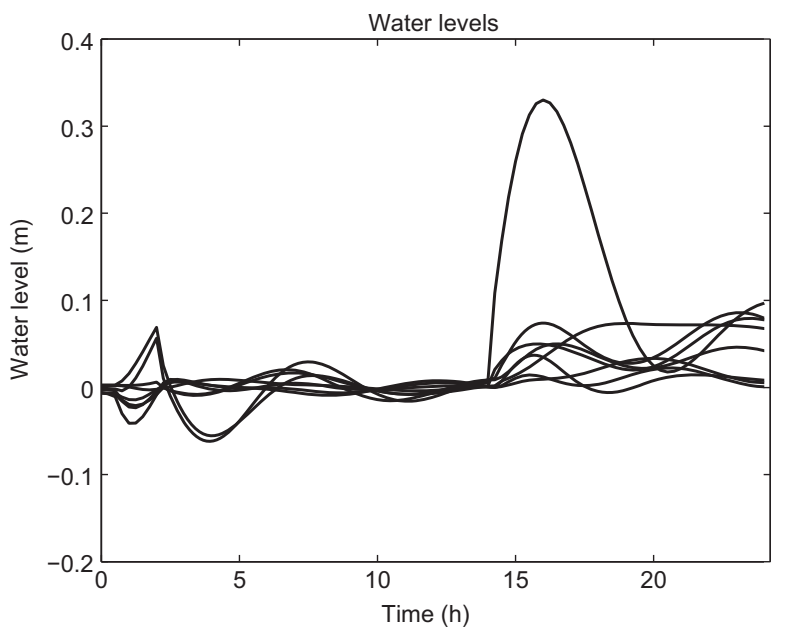

Fig. 15. ASCE Test Canal 2, Test 1: (a) MPC-B and (b) MPC-OF.

a

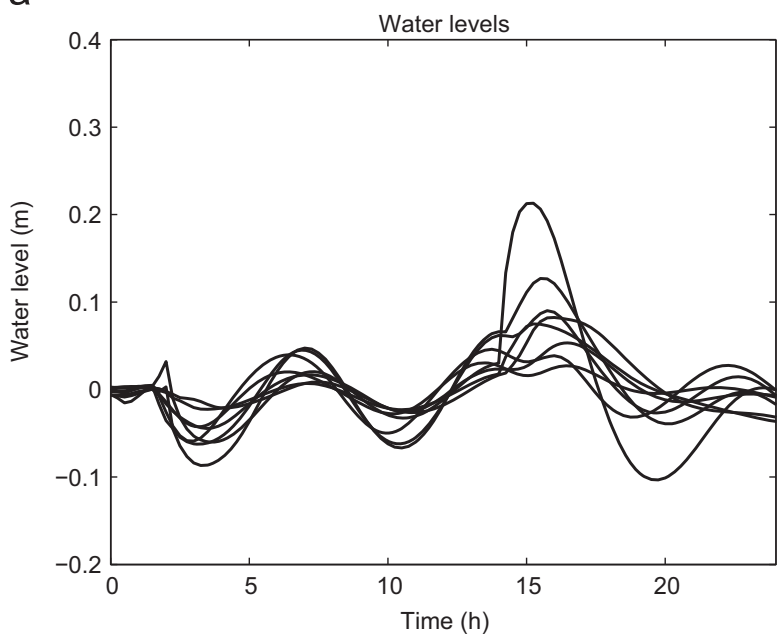

b

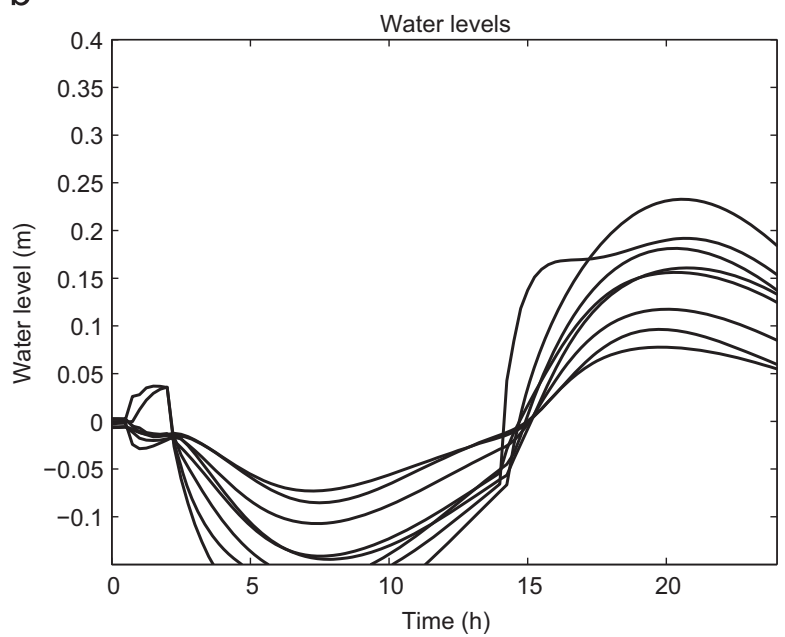

Fig. 16. ASCE Test Canal 2, Test 1 : (a) MPC-O and (b) MPC-I.

or using a different model. In the case of the ASCE Test Canal 2, the Integrator Delay model was used, so the resulting state space model was observable. The prediction horizon was set to 15 samples $(\lambda=15)$ for the numerical tests. Similar to the experimental tests, a sampling period of $10 \mathrm{~s}$ was used and the gates were adjusted at every $10 \mathrm{~s}$. The numerical test were carried out by the SIC software (Malaterre, 2012).

The numerical results for the UPC-PAC including feedforward method (the changes are known beforehand for the controller) are shown in Table 5. While seeing the maximum and the integral error, the normal method and the offset-free method produces similar errors. However, in case of unknown changes (Table 6) the integral of the error and the steady state error values are better for the offset-free method. Also, in case of no feedforward component, the MPC-FB method performs the best, however its performance is not so good in case of known changes.

The results of the disturbance test are shown in Figs. 12-14. Fig. 11 shows the results of the MPC without offset free method (MPC-B). While it rejects very well the known disturbances, in the presence of unknown disturbances the setpoint is not reached. However, in case of MPC-OF (Fig. 12), the steady state error is zero in both known and unknown cases. The results of MPC-OF are similar to those of MPC-FB (Fig. 14) for the unknown disturbances. The feedforward effect of the MPC-OF can be observed in

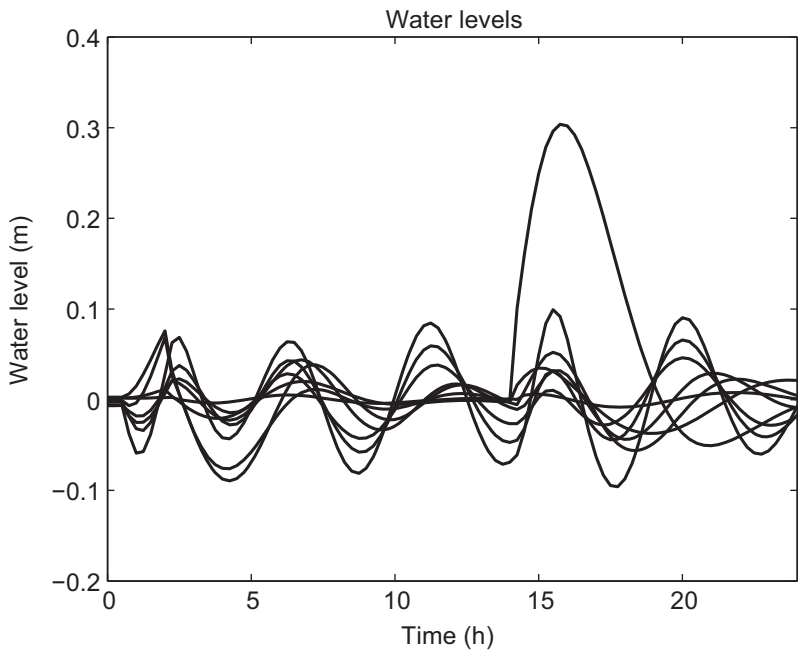

Fig. 17. ASCE Test canal 2, Test 1, MPC-FB.

Fig. 12a: the water level starts to increase before the actual disturbance occurs (noted with a dotted horizontal line). Fig. 13 shows the result of MPC-I: the water levels are reaching the 
Table 7

ASCE Canal 2, Test 1, Tuned.

\begin{tabular}{|c|c|c|c|c|c|c|c|c|c|c|c|c|}
\hline \multirow[t]{3}{*}{ Name } & \multicolumn{4}{|c|}{ MAE (\%) } & \multicolumn{4}{|c|}{ IAE (\%) } & \multicolumn{4}{|c|}{ StE (\%) } \\
\hline & \multicolumn{2}{|c|}{ 0-12 h } & \multicolumn{2}{|c|}{$12-24 \mathrm{~h}$} & \multicolumn{2}{|c|}{ 0-12 h } & \multicolumn{2}{|c|}{$12-24 \mathrm{~h}$} & \multicolumn{2}{|c|}{ 0-12 h } & \multicolumn{2}{|c|}{$12-24 \mathrm{~h}$} \\
\hline & Max. & Avg. & Max. & Avg. & Max. & Avg. & Max. & Avg. & Max. & Avg. & Max. & Avg. \\
\hline MPC & 4.7 & 2.1 & 14.7 & 4.8 & 0.8 & 0.5 & 10.5 & 2.7 & 0.3 & 0.2 & 12.5 & 2.8 \\
\hline MPC-OF & 4.1 & 1.7 & 19.4 & 5.0 & 1.2 & 0.5 & 6.8 & 2.2 & 0.7 & 0.3 & 4.2 & 2.3 \\
\hline MPC-O & 4.6 & 2.7 & 12.5 & 4.9 & 2.0 & 1.2 & 4.2 & 1.8 & 2.9 & 1.7 & 2.2 & 1.0 \\
\hline MPC-I & 11.2 & 7.5 & 12.2 & 8.2 & 7.6 & 4.8 & 8.9 & 5.6 & 8.4 & 5.6 & 11.5 & 7.6 \\
\hline MPC-FB & 4.7 & 2.7 & 17.9 & 4.5 & 2.0 & 1.1 & 5.4 & 1.7 & 2.8 & 1.0 & 2.2 & 1.2 \\
\hline
\end{tabular}

setpoint slowly after some initial oscillations. MPC-OF overperforms MPC-I, especially in Pool 3. However, for known disturbances MPC-OF shows an improvement.

Summarizing, the offset-free method (MPC-OF) balances between the handling of known and unknown disturbances, and shows good performance in both cases. While the MPC-B handles slightly better the known disturbances, and the MPC-FB handles equally the unknown disturbances, MPC-OF is able to handle both.

Disturbances can be known or unknown both in magnitude and in time. The work by Weyer (2008) discussed on the benefit of incorporating knowledge on future offtakes in LQ controllers and concluded that there is little benefit unless it is guaranteed that the offtake takes place at the scheduled time. This is an important practical issue. If a disturbance occurs at a different time that it was scheduled or predicted, then it has similar effects as an unknown disturbance occurring at a certain time. For example, recalling some results presented in Section 6.2, if there is a scheduled offtake of $20 \mathrm{l} / \mathrm{s}$ and it does not occur at the expected time, the controller will have the wrong information that the discharge has been reduced in $-20 \mathrm{l} / \mathrm{s}$. This can cause similar effects as if the offtake magnitude is not known. In any case, the controller derived in this study has the capability of reacting to the unexpected timing of the off take as if it was unknown.

\subsection{Numerical results on the ASCE benchmark Canal 2}

All five methods (MPC, MPC-FB, MPC-OF, MPC-O and MPC-I) have been numerically implemented on the ASCE Test Canal 2. The tuned Test 1 results are presented.

Figs. 15-17 show the results of the numerical tests. Fig. 15a shows the result of the MPC. All the water levels reach the setpoint in the first $12 \mathrm{~h}$ of the test, when the offtakes are known. However, during the second half of the test, some water levels are not at setpoint, there is a difference of $20 \mathrm{~cm}$. Using MPC-I (Fig. 16b), all the water levels are going to setpoint, however, they are going slowly, and the performance is clearly lower than from the case of MPC in the first half of the test. MPC-O has big oscillations in the first half of the test (Fig. 16b). MPC-OF arrives to no steady state error in the first half of the test, while in the second half it goes closed to the setpoint after a big overshoot (Fig. 15b). The same overshoot is seen in case of MPC-FB (Fig. 17), but for this controller there are constant oscillations during the first half of the test.

If the three controllers MPC (Fig. 15a), MPC-FB (Fig. 17) and MPC-OF are compared (Fig. 15b), it can be seen that MPC-OF balances between the performance of the two others: in the first half of the test it has slightly worse performance than MPC, but much better than MPC-FB, while in the second half of the test it goes much closer to the setpoint than MPC, but slightly less close than MPC-FB.

Table 7 shows the performance indicators calculated for the tuned Test 1 for the ASCE. The average of the MAE has the same order of magnitude for all the methods (except the MPC-I). In the case of the steady-state error for the first interval (that includes the known disturbances), the MPC method performs the best. The MPC-OF has slightly worst performance, while the other methods perform worse. In the second half of the test, MPC-O and MPC-FB method are the best, followed by the MPC-OF. It can be seen that the offset-free method is making a balance between providing good performance for both known and unknown changes.

\subsection{Influence of the tuning on the results}

It has been shown in the above sections that for these specific cases MPC-OF achieves better results than MPC-B or MPC-FB. However, as the performance of MPC depends on the tuning as well, to what extend this result might be caused by different tuning. First, it is clear that MPC-B cannot has steady state offset, and this cannot be eliminated with different tuning. This happens especially in cases when a gravity offtake is involved, and hence the controller has no clear knowledge of the outflow. For MPC-FB, the method has no knowledge of the future disturbances. This cannot be changed by tuning. In case of smaller disturbances, the difference between MPC-FB and MPC-OF might not be significant, as the knowledge about the disturbance will not improve the control. However, in case of larger known disturbances MPC-OF is expected to give better results.

As to the comparison with the other methods MPC-I and MPC$\mathrm{O}$, the results can clearly depend on the tuning. MPC-OF is not better than any of these other methods, here it was only shown that the results are comparable.

\section{Conclusions}

The main purpose of this study was to find a method to eliminate the offset of a predictive controller for water level in open channels. The method is based on a balanced combination of two strategies, one primarily suitable for control in the presence of unknown disturbances and another one including an integral effect able to cancel unknown disturbances.

It has been implemented to control water levels in a three-pool experimental canal and further numerically tested using a test case benchmark of a real long canal with eight controlled pools. Some experimental results indicate that this combined method in these cases can lead to better performance. In the test cases the method was able to achieve the desired water level set points in the case when the disturbances are known in advance as well in the case where no information is known about the disturbances, eliminating steady-state offsets. Further studies may be needed to confirm the validity of the proposed method for other cases. 


\section{References}

Aguilar, J., Langarita, P., Linares, L., \& Rodellar, J. (2009). Automatic control of flows and levels in an irrigation canal. IEEE Transactions on Industry Applications, 45, 2198-2208.

Aguilar, J. V., Langarita, P., Linares, L., Rodellar, J., \& Soler, J. (2012). Adaptive predictive expert control of levels in large canals for irrigation water distribution. International Journal of Adaptive Control and Signal Processing, 10, 945-960.

Badgwell, T., \& Muske, K. (2002). Disturbance model design for linear model predictive control. In Proceedings of the 2002 American control conference (pp. 1621-1626). IEEE.

Begovich, O., Ruiz, V. M., Besançon, G., Aldana, C., \& Georges, D. (2007). Predictive control with constraints of a multi-pool irrigation canal prototype. Latin American Applied Research, 37, 177-185.

Borrelli, F., \& Morari, M. (2007). Offset free model predictive control. In Proceedings of the 46th IEEE conference on decision and control. New Orleans, LA, USA.

Bryson, A. (1975). Applied optimal control: Optimization, estimation and control. Halsted Press Book, Taylor \& Francis.

Camacho, E. F., \& Bordons, C. (1998). Model predictive control. London: Springer.

Cantoni, M., Weyer, E., Li, Y., Ooi, S., Mareels, I., \& Ryan, M. (2007). Control of largescale irrigation networks. Proceedings of the IEEE, 95, 75-91.

Clemmens, A., Kacerek, T., Grawitz, B., \& Schuurmans, W. (1998). Test cases for canal control algorithms. Journal of Irrigation and Drainage Engineering, 124, 23-30.

Gómez, M., Rodellar, J., \& Mantecón, J. (2002). Predictive control method for decentralized operation of irrigation canals. Applied Mathematical Modelling, 26, 1039-1056.

Horváth, K. (2013). Model predictive control of resonance sensitive irrigation canals (Ph.D. thesis). Technical University of Catalonia. Barcelona, Spain.

Igreja, J., Cadete, F., \& Lemos, J. (2011). Application of distributed model predictive control to a water delivery canal. In 19th Mediterranean conference on control automation (MED) (pp. 682-687), Corfu, Greece.

Lemos, J. M., Machado, F., Nogueira, N., Rato, L. M., \& Rijo, M. (2009). Adaptive and non-adaptive model predictive control of an irrigation channel. Networks and Heterogeneous Media, 4, 303-324.

Malaterre, P. O. (2012). Sic 5.26, Simulation of irrigation canals. 〈http://sic.g-eau.net/ ?lang=fr $\rangle$. Accessed: 20/07/2021.

Malaterre, P. O., \& Baume, J. P. (1999). Optimum choice of control action variables and linked algorithms: Comparison of different alternatives. In Proceedings of USCID workshop on modernization of irrigation water delivery systems. Phoenix, AZ, USA (pp. 387-405)

Martín Sánchez, J. M., \& Rodellar, J. (1996). Adaptive predictive control: From the concepts to plant optimization. London: Prentice-Hall.

Mathworks, 2008. Matlab. 〈http://www.mathworks.com/products/matlab/〉.

Mosca, E. (1995). Optimal, predictive, and adaptive control. Englewood Cliffs, NJ: Prentice-Hall.
Negenborn, R., van Overloop, P. J., Keviczky, T., \& de Schutter, B. (2009). Distributed model predictive control of irrigation canals. Networks and Heterogeneous Media, 4, 359-380.

van Overloop, P. J. (2006). Model predictive control on open water systems (Ph.D. thesis). Delft University of Technology. Delft, The Netherlands.

van Overloop, P. J. Clemmens, A Strand, R. Wagemaker, R \& Bautista, E. (2010a). Real-time implementation of model predictive control on Maricopa-Stanfield irrigation and drainage district's WM canal. Journal of Irrigation and Drainage Engineering, 136, 747-756.

van Overloop, P. J., Horváth, K., \& Aydin, B. E. (2014). Model predictive control based on an integrator resonance model applied to an open water channel. Control Engineering Practice, 27, 54-60.

van Overloop, P. J., Miltenburg, I. J., Bombois, X., Clemmens, A. J., Strand, R., \& van de Giesen, N. (2010b). Identification of resonance waves in open water channels. Control Engineering Practice, 18, 863-872.

Pannocchia, G., \& Rawlings, J. B. (2003). Disturbance models for offset-free modelpredictive control. AIChE Journal, 49, 426-437.

Rodellar, J., Gómez, M., \& Bonet, L. (1993). Control method for on-demand operation of open channel flow. Journal of Irrigation and Drainage Engineering, $119,225-241$.

Rodellar, J., Gómez, M., \& Martín Vide, J. P. (1989). Stable predictive control of openchannel flow. Journal of Irrigation and Drainage Engineering, 115, 701-713.

Schuurmans, J., Bosgra, O., \& Brouwer, R. (1995). Open-channel flow model approximation for controller design. Applied Mathematical Modelling, 19, $525-530$

Sepúlveda, C. (2008). Instrumentation, model identification and control of an experimental irrigation canal (Ph.D. thesis). Technical University of Catalonia. Barcelona, Spain.

Venkat, A. N., Rawlings, J. B., \& Wright, S. J. (2006). Implementable distributed model predictive control with guaranteed performance properties. In: American control conference (pp. 613-618).

Wang, L. (2001). Use of exponential data weighting in model predictive control design. In: 2001. Proceedings of the 40th IEEE conference on decision and control (pp. 4857-4862).

Wang, L. (2009). Model predictive control system design and implementation using MATLAB. New York: Springer.

Weyer, E. (2001). System identification of an open water channel. Control Engineering Practice, 9, 1289-1299.

Weyer, E. (2008). Control of irrigation channels. IEEE Transactions on Control Systems Technology, 16, 664-675.

Zafra-Cabeza, A., Maestre, J. M., Ridao, M. A., Camacho, E. F., \& Sánchez, L. (2011). Hierarchical distributed model predictive control for risk mitigation: An irrigation canal case study. In American control conference (ACC) (pp. 3172 -3177). 